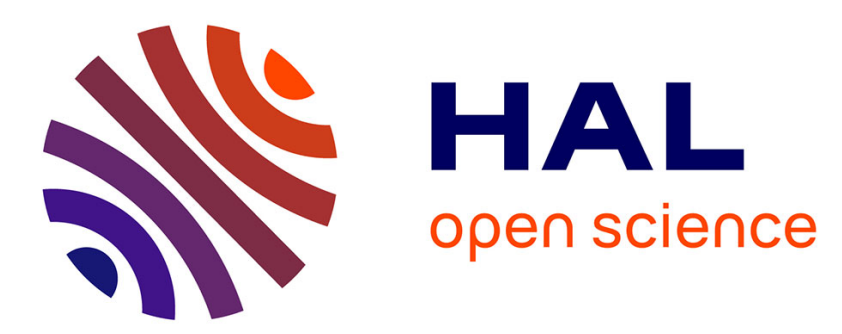

\title{
A variational formulation for the incremental homogenization of elasto-plastic composites
}

L. Brassart, Laurent Stainier, I. Doghri, L. Delannay

\section{To cite this version:}

L. Brassart, Laurent Stainier, I. Doghri, L. Delannay. A variational formulation for the incremental homogenization of elasto-plastic composites. Journal of the Mechanics and Physics of Solids, 2011, 59 (12), pp.2455-2475. 10.1016/j.jmps.2011.09.004 . hal-01004965

\section{HAL Id: hal-01004965 https://hal.science/hal-01004965}

Submitted on 24 Jun 2019

HAL is a multi-disciplinary open access archive for the deposit and dissemination of scientific research documents, whether they are published or not. The documents may come from teaching and research institutions in France or abroad, or from public or private research centers.
L'archive ouverte pluridisciplinaire HAL, est destinée au dépôt et à la diffusion de documents scientifiques de niveau recherche, publiés ou non, émanant des établissements d'enseignement et de recherche français ou étrangers, des laboratoires publics ou privés. 


\title{
A variational formulation for the incremental homogenization of elasto-plastic composites
}

\author{
L. Brassart ${ }^{\text {a }}$, L. Stainier ${ }^{\text {b }}$, I. Doghri ${ }^{\text {a }}$, L. Delannay ${ }^{\text {a }}$ \\ a Université catholique de Louvain, iMMC, 4 Av. Georges Lemaître, B-1348 Louvain-la-Neuve, Belgium \\ ${ }^{\mathrm{b}}$ Ecole Centrale de Nantes, GeM (UMR 6183 CNRS), 1 Rue de la Noë, BP 92101, F-44321 Nantes, France
}

This work addresses the micro-macro modeling of composites having elasto-plastic constituents. A new model is proposed to compute the effective stress-strain relation along arbitrary loading paths. The proposed model is based on an incremental variational principle (Ortiz, M., Stainier, L., 1999. The variational formulation of viscoplastic constitutive updates. Comput. Methods Appl. Mech. Eng. 171, 419-444) according to which the local stress-strain relation derives from a single incremental potential at each time step. The effective incremental potential of the composite is then estimated based on a linear comparison composite (LCC) with an effective behavior computed using available schemes in linear elasticity. Algorithmic elegance of the timeintegration of $\mathrm{J}_{2}$ elasto-plasticity is exploited in order to define the LCC. In particular, the elastic predictor strain is used explicitly. The method yields a homogenized yield criterion and radial return equation for each phase, as well as a homogenized plastic flow rule. The predictive capabilities of the proposed method are assessed against reference full-field finite element results for several particle-reinforced composites.
Keywords:

Micromechanics

Plasticity

Heterogeneous media

Particle-reinforced composites

\section{Introduction}

The prediction of the effective behavior of composite materials with elasto-plastic components is efficiently addressed by micromechanical approaches. According to the latter, the macroscopic mechanical response is defined as the relation between volume averages of stress and strain fields at the lower scale. Such modeling explicitly accounts for internal stresses which in turn affect the overall (anisotropic) yield surface and hardening. This paper focuses on the development of a semi-analytical homogenization model suitable for large-scale simulations of composite parts and structures.

In the linear elastic regime, the effective stress-strain relation is fully characterized by the overall stiffness tensor, to be computed once and for all from the elastic constants of the components given a statistical description of the microstructure. The problem can be equivalently restated into that of determining per-phase averages of the stress (or equivalently strain) field. Among well-known schemes one may cite Hashin-Shtrikman bounds (Hashin and Shtrikman, 1963; Willis, 1977), or the self-consistent (Kröner, 1958; Hill, 1965b) and Mori-Tanaka (Mori and Tanaka, 1973; Benveniste, 1987) approximations. 
In contrast, when plastic deformation develops, mechanical properties do not remain homogeneous within phases, and the local behavior becomes stress-dependent and history-dependent. Consequently, localization models valid in linear elasticity can no longer be applied. A workaround consists in considering a uniform plastic strain for each phase. The problem can then be handled within the framework of the transformation field analysis (TFA) (Dvorak and Benveniste, 1992; Dvorak, 1992) and elastic localization rules are applicable, considering the plastic strain as a (given) eigenstrain. Updates of the plastic strain are computed at each time step from the constitutive equations of the phase using the first moment of the stress within the phase. However, the method yields too stiff predictions (Suquet, 1997; Chaboche et al., 2001, 2005) unless each plastic region is subdivided into sub-domains in order to capture plastic strain heterogeneities, which obviously increases the model complexity. Still treating the plastic strain as an eigenstrain, Buryachenko (1999) developed alternative elastic interaction laws based on a statistical approach, and used second moments of the stress to evaluate the yield condition in each phase.

An alternative strategy consists in linearizing the local stress-strain relation around some reference state (usually chosen as the average deformation in the phase). This defines instantaneous, uniform properties for each phase. In the incremental method of Hill (1965a), tangent operators are used for the localization step in order to account for plastic accommodation in the redistribution of the stress and strain (increments) among the phases (see also Hutchinson, 1970; Turner and Tomé, 1994). Following Hill, other formulations based on linearized stress-strain relations were proposed, such as secant methods (Berveiller and Zaoui, 1979; Tandon and Weng, 1988) and non-incremental tangent formulations for viscoplasticity (Hutchinson, 1976; Molinari et al., 1987; Lebensohn and Tomé, 1993). An affine formulation for rateindependent elasto-plasticity was also proposed by Masson et al. (2000). Hill's incremental formulation is particularly well suited for elasto-plasticity, as it preserves the incremental structure of the constitutive equations at both phase and macroscopic levels. On the contrary, secant methods apply to plastic behavior only within a total deformation formalism.

It is well-recognized that both classical tangent and secant formulations yield too stiff responses (Gilormini, 1995; Suquet, 1996, 1997), and may even violate rigorous bounds obtained in the context of nonlinear elasticity by variational approaches, like those of Ponte Castañeda (1991). The reason for the overestimation might be attributed to the use of uniform linearized properties for each plastic phase. This observation motivated the development of methods which use second moments of the stress (or strain) to account for field fluctuations within the phases. A modified secant theory was proposed by Suquet (1995), which actually coincides with the variational procedure of Ponte Castañeda (1991) (Suquet, 1995; Ponte Castañeda and Suquet, 1998). In the context of incremental tangent methods, overly stiff predictions may be avoided by defining the linearized properties using isotropic tangent moduli, instead of anisotropic ones (González and LLorca, 2000; Doghri and Ouaar, 2003). This heuristic approach provides accurate predictions in many cases (Doghri and Friebel, 2005; Chaboche et al., 2005; Pierard et al., 2007). Some theoretical justifications for the use of isotropized tangent operators are found in Chaboche and Kanouté (2003), Chaboche et al. (2005), and Pierard and Doghri (2006).

Recently, Lahellec and Suquet (2007a,b) proposed an incremental variational formulation for materials with a hereditary behavior described by two potentials: a free energy and a dissipation function. It exploits an incremental variational formulation for the local behavior (Ortiz and Stainier, 1999) according to which the stress can be derived from a single incremental pseudo-potential. The effective behavior of the heterogeneous medium is then estimated following a variational formulation of the homogenization problem at each time step. A linear comparison composite (LCC) is defined based on a linearization of the dissipation potential and the introduction of piecewise uniform, reference internal variables. The proposed linearizations inspire respectively from the variational procedure of Ponte Castañeda (1991) (Lahellec and Suquet, 2007a) and the second-order method of Ponte Castañeda (1996) (Lahellec and Suquet, 2007b). The approach seems very promising, although estimates were so far presented within the context of nonlinear viscoelasticity only.

The present work is also based on the incremental variational principle of Ortiz and Stainier (1999), but the adopted strategy to introduce the LCC is different from Lahellec and Suquet. The new procedure exploits the concept of trial strain involved in the return mapping algorithm of $\mathrm{J}_{2}$ elasto-plasticity. The paper is organized as follows. Section 2 is a short, original presentation of the incremental variational principle of Ortiz and Stainier (1999) applied to small strain elastoplasticity. In Section 3, the homogenization problem is formulated by adopting a variational formalism in a timediscretized setting. Section 4 proposes an alternative and yet equivalent formulation of the problem based on a LCC. The formulation suggests an original localization rule based on the trial state. Based on this representation, a simple estimate is proposed in Section 5. Applications to two-phase particulate composites with highly contrasted phase properties are presented in Section 6. The model provides satisfying predictions of the effective response in most cases, and is able to sustain cyclic loads.

Throughout the paper, Einstein's convention is used, with indices ranging from 1 to 3 , unless otherwise indicated. The products of tensors are expressed as $(\boldsymbol{A}: \boldsymbol{\sigma})_{i j}=A_{i j k l} \sigma_{l k},(\boldsymbol{\sigma}: \boldsymbol{\sigma})=\sigma_{i j} \sigma_{j i}$, and $(\boldsymbol{\sigma} \otimes \boldsymbol{\sigma})_{i j k l}=\sigma_{i j} \sigma_{k l}$. The symbols 1 and $\boldsymbol{I}$ stand for the second and symmetric fourth order identity tensors, respectively. The spherical and deviatoric operators $\boldsymbol{I}^{\mathrm{vol}}$ and $\boldsymbol{I}^{\mathrm{dev}}$ are given by

$$
\boldsymbol{I}^{\mathrm{vol}} \equiv \frac{1}{3} \mathbf{1} \otimes \mathbf{1}, \quad \boldsymbol{I}^{\mathrm{dev}} \equiv \boldsymbol{I}-\boldsymbol{I}^{\mathrm{vol}} .
$$

The von Mises measures of stress and strain are respectively given by

$$
\sigma_{e q}=\left(\frac{3}{2} \boldsymbol{s}: \boldsymbol{s}\right)^{1 / 2} \text { and } \varepsilon_{e q}=\left(\frac{2}{3} \boldsymbol{e}: \boldsymbol{e}\right)^{1 / 2} \text {, }
$$


where $\boldsymbol{s}$ and $\boldsymbol{e}$ denote the deviatoric parts of $\boldsymbol{\sigma}$ and $\boldsymbol{\varepsilon}$ :

$$
\boldsymbol{s}=\boldsymbol{I}^{\mathrm{dev}}: \boldsymbol{\sigma}, \quad \boldsymbol{e}=\boldsymbol{I}^{\mathrm{dev}}: \boldsymbol{\varepsilon} .
$$

\section{Local constitutive equations}

We focus on materials whose local response can be described by classical $\mathrm{J}_{2}$ elasto-plastic theory with isotropic hardening. The behavior is assumed to be rate-independent and thermal effects are not considered. The constitutive equations are written within the framework of Generalized Standard Media (Halphen and Nguyen, 1975; Germain et al., 1983), according to which state laws and complementary laws for the evolution of the internal variables respectively derive from a free energy and a dissipation function.

Time integration of the constitutive equations along a given path of applied deformation is performed according to an incremental variational principle. Numerous authors contributed to the development of variational principles equivalent to the incremental formulation of elasto-plasticity (e.g. Mialon, 1986; Comi et al., 1991; Martin et al., 1996; Carini, 1996; Ortiz and Stainier, 1999; Miehe, 2002). In particular, Ortiz and Stainier (1999) proposed a unified formulation providing updates for the internal variables in the general context of elasto-(visco)plasticity at finite strains. On a time interval, updates of internal variables are obtained from the minimization of a suitably chosen functional, involving the free energy and the dissipation function. A remarkable advantage of the formulation is that the minimized functional constitutes a unique potential for the stress. This feature is the cornerstone of the homogenization procedure proposed in the sequel.

This section gives an extensive description of the incremental variational principle of Ortiz and Stainier (1999) in the case of small strain elasto-plasticity. In particular, the classical radial return scheme is shown to derive from this principle.

\subsection{Thermodynamic framework}

Under the small displacement hypothesis, the (symmetric) total strain tensor $\varepsilon$ is classically decomposed into an elastic and a plastic part:

$$
\boldsymbol{\varepsilon}=\boldsymbol{\varepsilon}^{e}+\boldsymbol{\varepsilon}^{p}
$$

The chosen set of state variables comprises the total strain $\boldsymbol{\varepsilon}$, the plastic strain $\boldsymbol{\varepsilon}^{p}$ and an additional scalar variable $p$ describing isotropic hardening and related to the accumulation of plastic deformation.

Contrarily to classical formulations (as described for instance in Lemaître and Chaboche, 1990; Maugin, 1992), no yield function is explicitly introduced. Instead, kinematic restrictions related to the plastic flow are postulated a priori. Based on the expected plastic flow kinematics in von Mises plasticity, the rate of plastic strain is split into a direction $\mathbf{N}$ and amplitude $\dot{p}$ :

$$
\dot{\boldsymbol{\varepsilon}}^{p}=\dot{p} \boldsymbol{N},
$$

where $\boldsymbol{N}$ is a kinematic variable ${ }^{1}$ satisfying the following constraints:

$$
\operatorname{tr}(\boldsymbol{N})=0 \text { and } \boldsymbol{N}: \boldsymbol{N}=\frac{3}{2} \text {. }
$$

The constraints on $\boldsymbol{N}$ ensure incompressibility of the plastic flow and uniqueness of decomposition (5). With such norm of $\boldsymbol{N}$, it is easy to check that: $\dot{p}=\left((2 / 3) \dot{\boldsymbol{\varepsilon}}^{p}: \dot{\boldsymbol{\varepsilon}}^{p}\right)^{1 / 2}$, so that the scalar variable $p$ is the classical accumulated plastic strain. The kinematic variable $\boldsymbol{N}$ will be specified later.

Supposing that the elastic response is independent of irreversible processes, the Helmholtz free energy (per unit volume) admits the following additive decomposition:

$$
\psi\left(\boldsymbol{\varepsilon}, \boldsymbol{\varepsilon}^{p}, p\right)=\psi^{e}\left(\boldsymbol{\varepsilon}-\boldsymbol{\varepsilon}^{p}\right)+\psi^{p}(p)
$$

The elastic part $\psi^{e}$ represents the energy stored within the material and recoverable through elastic relaxation. A linear response in the elastic regime is obtained by taking $\psi^{e}$ quadratic in the elastic strain:

$$
\psi^{e}\left(\boldsymbol{\varepsilon}-\boldsymbol{\varepsilon}^{p}\right)=\frac{1}{2}\left(\boldsymbol{\varepsilon}-\boldsymbol{\varepsilon}^{p}\right): \boldsymbol{C}^{e}:\left(\boldsymbol{\varepsilon}-\boldsymbol{\varepsilon}^{p}\right)
$$

where $\boldsymbol{C}^{e}$ is the elastic stiffness operator. The plastic part $\psi^{p}$ describing (isotropic) hardening is written as

$$
\psi^{p}(p)=\int_{0}^{p} R(q) d q
$$

where $R(q)$ represents the hardening stress (the function $R$ is supposed to be given). Kinematic hardening can be modeled by including a dependence of the plastic potential on the plastic strain $\boldsymbol{\varepsilon}^{p}$. However, kinematic hardening is not considered in the homogenization model proposed here.

\footnotetext{
${ }^{1}$ The kinematic variable $\boldsymbol{N}$ represents the direction of plastic flow. It should not be confused with some kinematic variable for the description of kinematic hardening. The latter is not considered in the present work.
} 
The state law for the stress is obtained from the free energy as

$$
\boldsymbol{\sigma}=\frac{\partial \psi}{\partial \boldsymbol{\varepsilon}}\left(\boldsymbol{\varepsilon}, \boldsymbol{\varepsilon}^{p}, p\right)=-\frac{\partial \psi}{\partial \boldsymbol{\varepsilon}^{p}}\left(\boldsymbol{\varepsilon}, \boldsymbol{\varepsilon}^{p}, p\right)=\frac{\partial \psi^{e}}{\partial \boldsymbol{\varepsilon}^{e}}\left(\boldsymbol{\varepsilon}-\boldsymbol{\varepsilon}^{p}\right) .
$$

Hence, the stress is the force associated with both the total strain and the plastic strain. Similarly, the so-called hardening stress is the thermodynamic force associated with the internal variable $p$ :

$$
R=\frac{\partial \psi}{\partial p}\left(\boldsymbol{\varepsilon}, \boldsymbol{\varepsilon}^{p}, p\right)=\frac{\partial \psi^{p}}{\partial p}(p) .
$$

State laws (10) and (11) must be supplemented by a kinetic relation prescribing the evolution of the internal variable $p$ (the evolution of $\varepsilon^{p}$ being given by the flow rule (5)). The complementary law must ensure that the mechanical dissipation $\mathcal{D}$ is non-negative. Here, the dissipation expresses as (e.g. Lemaître and Chaboche, 1990):

$$
\mathcal{D}=\boldsymbol{\sigma}: \dot{\boldsymbol{\varepsilon}}^{p}-R \dot{p} \geq 0 .
$$

The dissipation may conveniently be rewritten in a condensed form that accounts for the flow rule (5):

$$
\mathcal{D}=Y(\boldsymbol{N}) \dot{p},
$$

where the function $Y$ is defined, for a given state $\left\{\varepsilon, \varepsilon^{p}, p\right\}$, as

$$
Y(\boldsymbol{N})=\boldsymbol{\sigma}: \boldsymbol{N}-R .
$$

Expression (13) of the dissipation indicates that the new scalar quantity $Y$ is the force conjugated to $p$, when it is computed for the actual flow direction $\boldsymbol{N}$. Then, the evolution law for $p$ can be expressed as a kinetic relation between $Y$ and $\dot{p}$. Based on the theory of Generalized Standard Media, it is supposed to derive from a dissipation function $\phi(\dot{p})$ :

$$
Y=\frac{\partial \phi}{\partial \dot{p}}(\dot{p}) \quad \text { or, equivalently } \dot{p}=\frac{\partial \phi^{*}}{\partial Y}(Y),
$$

where $\phi^{*}$ is the convex dual of $\phi$ by Legendre transform:

$$
\phi^{*}(Y)=\sup _{\dot{p}}\{\dot{p} Y-\phi(\dot{p})\} .
$$

By choosing $\phi(\dot{p})$ non-negative, convex and such that $\phi(0)=0$, the mechanical dissipation (13) is necessarily positive.

Remark 1. The comparison between $Y$ and a thermodynamic force can be further justified on energy basis. Consider virtual (and independent) perturbations $p \rightarrow p+\delta p$ and $\varepsilon^{p} \rightarrow \varepsilon^{p}+\delta \varepsilon^{p}$, with $\delta \varepsilon^{p} \equiv \tilde{\boldsymbol{N}} \delta p, \tilde{\boldsymbol{N}}$ being an arbitrary flow direction. The total deformation is kept constant. The corresponding variation of the free energy is then

$$
\delta \psi=\left(\frac{\partial \psi^{e}}{\partial \boldsymbol{\varepsilon}^{e}}: \frac{\partial \boldsymbol{\varepsilon}^{e}}{\partial \boldsymbol{\varepsilon}^{p}}: \delta \boldsymbol{\varepsilon}^{p}+\frac{\partial \psi^{p}}{\partial p} \delta p\right) \equiv-Y(\tilde{\boldsymbol{N}}) \delta p .
$$

Thus, the quantity $Y(\tilde{\boldsymbol{N}}) \delta p$ measures the variation of free energy at constant total deformation, for a given variation of $p$ and an arbitrary direction of plastic flow $\tilde{\boldsymbol{N}}$.

The classical equations of rate-independent elasto-plasticity are retrieved by taking the dissipation function homogeneous of degree one with respect to (w.r.t.) $\dot{p}$ :

$$
\phi(\dot{p})= \begin{cases}\sigma_{Y} \dot{p}, & \dot{p} \geq 0 \\ +\infty & \text { otherwise }\end{cases}
$$

Since $\phi$ is not differentiable at $\dot{p}=0$, the partial derivative in (15) must be understood in the sense of sub-differential (Rockafellar, 1970; Moreau, 1976). The kinetic relation (15) yields

$$
\begin{aligned}
& Y<\sigma_{Y} \Leftrightarrow \dot{p}=0, \\
& Y=\sigma_{Y} \Leftrightarrow \dot{p} \geq 0 .
\end{aligned}
$$

In other words, deformations are purely elastic when the forces are inside an elasticity domain $\left[0, \sigma_{Y}[\right.$. Plastic flow occurs when the force $Y$ reaches the yield stress $\sigma_{Y}$. In addition, $Y$ cannot leave the yield surface when plastic deformation occurs $(\dot{p}>0)$. On the other hand, negative values of $\dot{p}$ are prohibited, as they would imply infinite dissipation. The function $\phi(\dot{p})$ defines a convex set whose indicator function is precisely the dual $\phi^{*}$ of $\phi$ (see e.g. Maugin, 1992), which is here given by

$$
\phi^{*}(Y)= \begin{cases}0, & Y \leq \sigma_{Y} \\ +\infty & \text { otherwise. }\end{cases}
$$

This convex set precisely coincides with the elasticity domain just introduced. Thus, the existence of an elasticity domain follows from the definition of the dissipation function.

The kinematic variable $\boldsymbol{N}$ was not specified up to now. Actually, it can be shown that $\boldsymbol{N}$ is found by maximizing the dissipation at fixed $p$ and $\dot{p}$. This follows from a continuous variational principle introduced by Ortiz and Stainier (1999), 
which is not presented here for brevity. The kinematic variable will be specified within the discretized formulation presenter hereafter.

\subsection{Incremental variational principle}

We now consider the problem of integrating the constitutive relations over a time increment $\left[t_{n}, t_{n+1}\right]$. The state at $t_{n}$ is supposed to be given: $\left\{\boldsymbol{\varepsilon}_{n}, \boldsymbol{\varepsilon}_{n}^{p}, p_{n}\right\}$, so as the total deformation at $t_{n+1}: \boldsymbol{\varepsilon}_{n+1}$. We aim to compute the stress $\boldsymbol{\sigma}_{n+1}$, the plastic strain $\varepsilon_{n+1}^{p}$ and the accumulated plastic strain $p_{n+1}$ at $t_{n+1}$. We first assume that the rate of accumulated plastic strain $\dot{p}$ is constant over the time step and given by the ratio $\Delta p / \Delta t$, with $\Delta(\cdot)=(\cdot)_{n+1}-(\cdot)_{n}$. Similarly, the plastic flow rule (5) is discretized as

$$
\Delta \boldsymbol{\varepsilon}^{p}=\Delta p \boldsymbol{N}
$$

where $\boldsymbol{N}$ is an (a priori unknown) constant plastic flow direction for the time step. Ortiz and Stainier (1999) proposed the following incremental variational principle:

$$
W_{\Delta}\left(\boldsymbol{\varepsilon}_{n+1}\right)=\inf _{\Delta p, \boldsymbol{N}} J_{\Delta}\left(\boldsymbol{\varepsilon}_{n+1}, \Delta p, \boldsymbol{N}\right),
$$

where the minimization w.r.t. $\boldsymbol{N}$ is performed under constraints (6) and

$$
J_{\Delta}\left(\boldsymbol{\varepsilon}_{n+1}, \Delta p, \boldsymbol{N}\right)=\psi\left(\boldsymbol{\varepsilon}_{n+1}, \boldsymbol{\varepsilon}_{n+1}^{p}, p_{n+1}\right)-\psi_{n}+\Delta t \phi\left(\frac{\Delta p}{\Delta t}\right),
$$

where $\varepsilon_{n+1}^{p}$ is obtained from the discretized flow rule (21) and $\psi_{n}$ is the free energy computed for the (given) state variables at $t_{n}$. Then, considering the stationarity conditions w.r.t. $\Delta p$ and $\boldsymbol{N}$ in (22), the stress tensor at $t_{n+1}$ is given by

$$
\boldsymbol{\sigma}_{n+1}=\frac{d W_{\Delta}}{d \boldsymbol{\varepsilon}_{n+1}}\left(\boldsymbol{\varepsilon}_{n+1}\right)=\frac{\partial J_{\Delta}}{\partial \boldsymbol{\varepsilon}_{n+1}}\left(\boldsymbol{\varepsilon}_{n+1}, \Delta p, \boldsymbol{N}\right),
$$

where $\Delta p$ and $\boldsymbol{N}$ are the solutions of the minimization problem (22). Thus, the function $W_{\Delta}$ plays the role of an incremental potential for the stress. In the following we show that the optimality conditions w.r.t. $\Delta p$ and $\boldsymbol{N}$ yield the classical incremental relations of $\mathrm{J}_{2}$ plasticity. In particular, the well-known radial return scheme with its predictor and corrector steps (Wilkins, 1964, see also Simo and Hughes, 1998 or Doghri, 2000) is retrieved.

Taking into account the discretized flow rule (21), the stationarity condition of $J_{\Delta}$ w.r.t. $\Delta p$ gives the discretized kinetic relation (15):

$$
Y_{n+1}\left(\boldsymbol{N}, p_{n+1}\right)=\frac{\partial \phi}{\partial \dot{p}}\left(\frac{\Delta p}{\Delta t}\right)
$$

where the function $Y_{n+1}$ is defined similarly as in the continuous case (14):

$$
Y_{n+1}\left(\boldsymbol{N}, p_{n+1}\right) \equiv \boldsymbol{\sigma}_{n+1}: \boldsymbol{N}-R\left(p_{n+1}\right) .
$$

Note that $\boldsymbol{\sigma}_{n+1}$ now depends on $\boldsymbol{N}$, since:

$$
\boldsymbol{\sigma}_{n+1}=\frac{\partial \psi^{e}}{\partial \boldsymbol{\varepsilon}^{e}}\left(\boldsymbol{\varepsilon}_{n+1}^{e}\right) \quad \text { with } \boldsymbol{\varepsilon}_{n+1}^{e}=\boldsymbol{\varepsilon}_{n+1}-\boldsymbol{\varepsilon}_{n}^{p}-\Delta p \boldsymbol{N}
$$

Therefore, it is conveniently rewritten as

$$
\boldsymbol{\sigma}_{n+1}=\boldsymbol{C}^{e}:\left(\boldsymbol{\varepsilon}_{n+1}^{t r}-\Delta p \boldsymbol{N}\right)=\boldsymbol{\sigma}_{n+1}^{t r}-\Delta p\left(\boldsymbol{C}^{e}: \boldsymbol{N}\right),
$$

introducing the trial (or predictor) elastic strain $\varepsilon_{n+1}^{\text {tr }}$ and the corresponding trial stress:

$$
\begin{aligned}
& \boldsymbol{\varepsilon}_{n+1}^{t r} \equiv \boldsymbol{\varepsilon}_{n+1}-\boldsymbol{\varepsilon}_{n}^{p}, \\
& \boldsymbol{\sigma}_{n+1}^{t r} \equiv \boldsymbol{C}^{e}: \boldsymbol{\varepsilon}_{n+1}^{t r} .
\end{aligned}
$$

The minimization of $J_{\Delta}$ w.r.t. $\boldsymbol{N}$ under constraints (6) is performed using Lagrange multipliers and it yields (see Appendix A)

$$
\boldsymbol{N}=\frac{3}{2} \frac{\boldsymbol{s}_{n+1}^{t r}}{\sigma_{e q, n+1}^{t r}}=\frac{\boldsymbol{e}^{t r}}{\varepsilon_{e q}^{t r}} .
$$

Expression (31) of the kinematic variable is obtained assuming isotropic elasticity, in which case the elastic stiffness tensor admits the following decomposition:

$$
\boldsymbol{C}^{e}=3 \kappa \boldsymbol{I}^{\mathrm{vol}}+2 \mu \boldsymbol{I}^{\mathrm{dev}},
$$

where $\kappa$ and $\mu$ are the elastic bulk and shear moduli, respectively.

Substituting (31) into (26), the stationarity condition (25) for $\Delta p$ becomes

$$
-3 \mu \varepsilon_{e q, n+1}^{t r}+3 \mu \Delta p+R\left(p_{n+1}\right)+\frac{\partial \phi}{\partial \dot{p}}\left(\frac{\Delta p}{\Delta t}\right)=0 .
$$


The problem of the non-smoothness of the dissipation function for $\Delta p=0$ can be circumvented by first evaluating the slope of the functional $J_{\Delta}$ for $\Delta p=0^{+}$. If it is negative, that is

$$
-3 \mu \varepsilon_{e q, n+1}^{t r}+R\left(p_{n}\right)+\sigma_{Y}<0,
$$

then the optimal $\Delta p$ is positive, and satisfies the following equation:

$$
-3 \mu \varepsilon_{e q, n+1}^{t r}+3 \mu \Delta p+R\left(p_{n}+\Delta p\right)+\sigma_{Y}=0 .
$$

Otherwise, the optimal $\Delta p$ is zero, as negative values are prohibited (they would lead to infinite dissipation), and the increment is elastic. Therefore, the minimization problem associated with the incremental variational principle involves the evaluation of a yield criterion in terms of an elastic predictor, and a plastic correction step, exactly like in the classical return mapping.

Remark 2. The incremental functionals $W_{\Delta}$ and $J_{\Delta}$ are related to a time increment and they depend on the past loading history. Therefore, they are not state functions. For this reason, $W_{\Delta}$ should preferably be referred to as a pseudo-potential for the stress.

\section{Homogenization}

\subsection{Local potential}

We consider a representative volume element (RVE) $V$ of a composite with $N$ elasto-plastic phases $r=1, \ldots, N$. Each phase occupies a domain $V_{r}$ of the RVE with volume fraction $c_{r}=V_{r} / V$ and characteristic function $\chi^{(r)}$, with $\chi^{(r)}(\boldsymbol{x})=1$ if $\boldsymbol{x}$ is in phase $r$, and 0 elsewhere. The local constitutive behavior is characterized by a free energy and a dissipation function:

$$
\psi\left(\boldsymbol{x}, \boldsymbol{\varepsilon}, \boldsymbol{\varepsilon}^{p}, p\right)=\sum_{r=1}^{N} \chi^{(r)}(\boldsymbol{x}) \psi^{(r)}\left(\boldsymbol{\varepsilon}, \boldsymbol{\varepsilon}^{p}, p\right), \quad \phi(\boldsymbol{x}, \dot{p})=\sum_{r=1}^{N} \chi^{(r)}(\boldsymbol{x}) \phi^{(r)}(\dot{p}),
$$

where

$$
\begin{aligned}
& \psi^{(r)}\left(\boldsymbol{\varepsilon}, \boldsymbol{\varepsilon}^{p}, p\right)=\psi^{e(r)}\left(\boldsymbol{\varepsilon}-\boldsymbol{\varepsilon}^{p}\right)+\psi^{p(r)}(p), \\
& \psi^{e(r)}\left(\boldsymbol{\varepsilon}-\boldsymbol{\varepsilon}^{p}\right)=\frac{1}{2}\left(\boldsymbol{\varepsilon}-\boldsymbol{\varepsilon}^{p}\right): \boldsymbol{C}^{e(r)}:\left(\boldsymbol{\varepsilon}-\boldsymbol{\varepsilon}^{p}\right), \\
& \boldsymbol{C}^{e(r)}=3 \kappa^{(r)} \boldsymbol{I}^{\mathrm{vol}}+2 \mu^{(r)} \mathbf{I}^{\mathrm{dev}}, \\
& \psi^{p(r)}(p)=\int_{0}^{p} R^{(r)}(q) d q, \\
& \phi^{(r)}(\dot{p})=\dot{p} \sigma_{Y}^{(r)} \text { if } \dot{p} \geq 0, \quad+\infty \text { otherwise. }
\end{aligned}
$$

Adopting the incremental setting of Section 2.2, and assuming the local state at $t_{n}$ to be given, the internal variables $\varepsilon_{n+1}^{p}$ and $p_{n+1}$, as well as the kinematic variable $\boldsymbol{N}$ are determined from the solution of the following local minimization problem at a given material point:

$$
W_{\Delta}\left(\boldsymbol{x}, \boldsymbol{\varepsilon}_{n+1}\right)=\inf _{\Delta p, \boldsymbol{N}} J_{\Delta}\left(\boldsymbol{x}, \boldsymbol{\varepsilon}_{n+1}, \Delta p, \boldsymbol{N}\right),
$$

where the minimization w.r.t. $\boldsymbol{N}$ is performed under constraints (6). The functional $J_{\Delta}$ is given by

$$
J_{\Delta}\left(\boldsymbol{x}, \boldsymbol{\varepsilon}_{n+1}, \Delta p, \boldsymbol{N}\right)=\psi\left(\boldsymbol{x}, \boldsymbol{\varepsilon}_{n+1}, \boldsymbol{\varepsilon}_{n+1}^{p}, p_{n+1}\right)-\psi_{n}(\boldsymbol{x})+\Delta t \phi\left(\boldsymbol{x}, \frac{\Delta p}{\Delta t}\right),
$$

where $\boldsymbol{\varepsilon}_{n+1}^{p}$ depends on $\Delta p$ and $\boldsymbol{N}$ through the incremental flow rule (21). Note that $J_{\Delta}$ (and thus $W_{\Delta}$ ) depend on $\boldsymbol{x}$ not only through the characteristic functions $\chi^{(r)}$ in (36), but also through the fields $\varepsilon_{n}^{p}(\boldsymbol{x})$ and $p_{n}(\boldsymbol{x})$. The function (42) acts as a potential for the stress:

$$
\boldsymbol{\sigma}_{n+1}=\frac{\partial W_{\Delta}}{\partial \boldsymbol{\varepsilon}_{n+1}}\left(\boldsymbol{\varepsilon}_{n+1}\right)
$$

\subsection{Effective behavior}

Let $\langle\cdot\rangle$ and $\langle\cdot\rangle_{r}$ denote a volume average over the RVE and phase $r$, respectively, with $\langle\cdot\rangle_{=}=\sum_{r=1}^{N} c_{r}\langle\cdot\rangle_{r}$. We aim to compute the effective stress response of the composite $\bar{\sigma}(t) \equiv\langle\boldsymbol{\sigma}(t)\rangle$ for a given history of prescribed deformation $\overline{\boldsymbol{\varepsilon}}(t) \equiv\langle\boldsymbol{\varepsilon}(t)\rangle$. In the time-discretized setting, the effective behavior of the composite at time $t_{n+1}$ can be determined from the effective incremental energy function (Miehe, 2002; Lahellec and Suquet, 2007a):

$$
\bar{W}_{\Delta}\left(\overline{\boldsymbol{\varepsilon}}_{n+1}\right) \equiv \inf _{\varepsilon_{n+1} \in \mathcal{K}\left(\overline{\boldsymbol{\varepsilon}}_{n+1}\right)}\left\langle W_{\Delta}\left(\boldsymbol{x}, \boldsymbol{\varepsilon}_{n+1}\right)\right\rangle,
$$


where the set $\mathcal{K}\left(\overline{\boldsymbol{\varepsilon}}_{n+1}\right)$ of admissible strain fields is defined as

$$
\mathcal{K}\left(\overline{\boldsymbol{\varepsilon}}_{n+1}\right)=\left\{\boldsymbol{\varepsilon}_{n+1}=\frac{1}{2}\left((\boldsymbol{\nabla u})_{n+1}+(\boldsymbol{\nabla u})_{n+1}^{T}\right),\left\langle\boldsymbol{\varepsilon}_{n+1}\right\rangle=\overline{\boldsymbol{\varepsilon}}_{n+1}\right\},
$$

where $\boldsymbol{u}$ denotes the displacement field within the RVE. For definiteness of problem (45), boundary conditions satisfying the constraint on the average of the strain field in (46) must be imposed on the boundary of the RVE. Linear displacement boundary conditions such that $\boldsymbol{u}_{n+1}=\overline{\boldsymbol{\varepsilon}}_{n+1} \cdot \boldsymbol{x}$ on $\partial V$ may be adopted. Periodic boundary conditions or surface traction boundary conditions could alternatively be considered, see for instance Miehe (2002).

Eq. (45) defines an effective incremental potential for the composite $\bar{W}_{\Delta}$ from which the macroscopic stress is derived:

$$
\overline{\boldsymbol{\sigma}}_{n+1}=\left\langle\boldsymbol{\sigma}_{n+1}\right\rangle=\frac{\partial \bar{W}_{\Delta}}{\partial \overline{\boldsymbol{\varepsilon}}_{n+1}}\left(\overline{\boldsymbol{\varepsilon}}_{n+1}\right) \text {. }
$$

A proof of the latter equality is given by Lahellec and Suquet (2007a) and reads as follows. First, the derivative of $\bar{W}_{\Delta}$ w.r.t. $\overline{\boldsymbol{\varepsilon}}_{n+1}$ is computed:

$$
\frac{\partial \bar{W}_{\Delta}}{\partial \overline{\boldsymbol{\varepsilon}}_{n+1}}\left(\overline{\boldsymbol{\varepsilon}}_{n+1}\right)=\left\langle\frac{\partial J_{\Delta}}{\partial \boldsymbol{\varepsilon}_{n+1}}\left(\boldsymbol{x}, \boldsymbol{\varepsilon}_{n+1}, \boldsymbol{\alpha}_{n+1}\right): \frac{\partial \boldsymbol{\varepsilon}_{n+1}}{\partial \overline{\boldsymbol{\varepsilon}}_{n+1}}\right\rangle+\left\langle\frac{\partial J_{\Delta}}{\partial \boldsymbol{\alpha}_{n+1}}\left(\boldsymbol{x}, \boldsymbol{\varepsilon}_{n+1}, \boldsymbol{\alpha}_{n+1}\right): \frac{\partial \boldsymbol{\alpha}_{n+1}}{\partial \overline{\boldsymbol{\varepsilon}}_{n+1}}\right\rangle,
$$

where $\boldsymbol{\alpha}_{n+1}$ collectively denotes $\Delta p$ and $\boldsymbol{N}$, solutions of the minimization problem (42) at $t_{n+1}$. The second term on the right vanishes due to the stationarity of $J_{\Delta}$ w.r.t. $\boldsymbol{\alpha}_{n+1}$ (implicitly taking the kinematic constraints on $\boldsymbol{N}$ into account) and the first one gives, thanks to Hill's Lemma (Hill, 1967):

$$
\left\langle\boldsymbol{\sigma}_{n+1}: \frac{\partial \boldsymbol{\varepsilon}_{n+1}}{\partial \overline{\boldsymbol{\varepsilon}}_{n+1}}\right\rangle=\left\langle\boldsymbol{\sigma}_{n+1}\right\rangle:\left\langle\frac{\partial \boldsymbol{\varepsilon}_{n+1}}{\partial \overline{\boldsymbol{\varepsilon}}_{n+1}}\right\rangle=\left\langle\boldsymbol{\sigma}_{n+1}\right\rangle=\overline{\boldsymbol{\sigma}}_{n+1} .
$$

Making use of expressions (36), (42) and (43), the effective incremental potential (45) is rewritten as

$$
\bar{W}_{\Delta}\left(\overline{\boldsymbol{\varepsilon}}_{n+1}\right)=\inf _{\boldsymbol{\varepsilon}_{n+1} \in \mathcal{K}\left(\overline{\boldsymbol{\varepsilon}}_{n+1}\right)}\left\langle\inf _{\Delta p, \boldsymbol{N}}\left\{\sum_{r=1}^{N} \chi^{(r)}(\boldsymbol{x})\left\{\psi^{(r)}\left(\boldsymbol{\varepsilon}_{n+1}, \boldsymbol{\varepsilon}_{n+1}^{p}, p_{n+1}\right)-\psi_{n}^{(r)}(\boldsymbol{x})+\Delta t \phi^{(r)}\left(\frac{\Delta p}{\Delta t}\right)\right\}\right\}\right\rangle .
$$

The solution fields $\Delta p$ and $\boldsymbol{N}$ fluctuate within the composite, depending on the local strain $\boldsymbol{\varepsilon}_{n+1}$ and state variables at $t_{n}, \boldsymbol{\varepsilon}_{n}^{p}$ and $p_{n}$. Obviously, an exact semi-analytical solution for problem (50) is out of range and approximations are required.

A straightforward simplification to problem (50) consists in considering piecewise uniform internal variables. As shown in Appendix B, such simplification leads to the transformation field analysis (TFA): the strain field is computed on a comparison composite characterized by the elastic moduli, with the plastic strain acting as an eigenstrain. Updates of the internal variables result from the incremental variational principle and obey a radial return scheme for each phase. However, it is well known that the predictions of the TFA are too stiff when it is applied to two-phase systems, because it is based on purely elastic accommodation.

The homogenization model presented in the sequel is based on different linearized interaction relations. Inspiring from the variational technique of Ponte Castañeda $(1991,1992)$, an original linearization strategy is presented, according to which the effective potential (50) is reexpressed in terms of the effective potential of a linear comparison composite (LCC) characterized by secant operators for the trial strain-stress relation. Based on this formulation, estimates of the effective behavior are proposed. In the remainder of the paper, all quantities are evaluated at $t_{n+1}$ (subscripts $(n+1)$ omitted for simplicity), unless otherwise indicated.

\section{Variational procedure: definition of a linear comparison composite}

\subsection{Phase potential}

As a starting point, we consider that the infimum over the kinematic variable $\boldsymbol{N}$ in (50) is satisfied at each material point in the composite, so that expression (31) may be used. Then, the elastic strain can be expressed as a function of the trial strain and $\Delta p$ as

$$
\boldsymbol{\varepsilon}^{e}=\boldsymbol{\varepsilon}^{t r}-\Delta \boldsymbol{\varepsilon}^{p}=\boldsymbol{\varepsilon}^{t r}-\Delta p \boldsymbol{N} \quad \text { with } \boldsymbol{N}=\frac{\boldsymbol{e}^{t r}}{\varepsilon_{e q}^{t r}} .
$$

It follows that the hydrostatic and equivalent elastic strains can be rewritten as

$$
\varepsilon_{m}^{e}=\varepsilon_{m}^{t r} \quad \text { and } \quad \varepsilon_{e q}^{e}=\left(1-\frac{\Delta p}{\varepsilon_{e q}^{t r}}\right) \varepsilon_{e q}^{t r}
$$

where the hydrostatic strain is defined as: $\varepsilon_{m} \equiv \frac{1}{3} \operatorname{tr}(\boldsymbol{\varepsilon})$. Now, consider the following expression for the elastic free energy (38):

$$
\psi^{e(r)}\left(\boldsymbol{\varepsilon}^{e}\right)=\frac{9}{2} \kappa^{(r)}\left(\varepsilon_{m}^{e}\right)^{2}+\frac{3}{2} \mu^{(r)}\left(\varepsilon_{e q}^{e}\right)^{2} .
$$


Introducing relations (52) into the latter expression, the elastic free energy can be reexpressed in terms of the trial strain. It yields

$$
\psi^{e(r)}\left(\varepsilon^{e}\right) \equiv \Psi^{e(r)}\left(\varepsilon^{t r}, \Delta p\right)=\frac{9}{2} \kappa^{(r)}\left(\varepsilon_{m}^{t r}\right)^{2}+f^{(r)}\left(\left(\varepsilon_{e q}^{t r}\right)^{2}, \Delta p\right),
$$

where the function $f^{(r)}$ is given by

$$
f^{(r)}\left(\left(\varepsilon_{e q}^{t r}\right)^{2}, \Delta p\right)=\frac{3}{2} \mu^{(r)}\left(1-\frac{\Delta p}{\sqrt{\left(\varepsilon_{e q}^{t r}\right)^{2}}}\right)^{2}\left(\varepsilon_{e q}^{t r}\right)^{2}=\frac{3}{2} \mu^{(r)}\left(\sqrt{\left(\varepsilon_{e q}^{t r}\right)^{2}}-\Delta p\right)^{2},
$$

which is a non-negative function of $\left(\varepsilon_{e q}^{t r}\right)^{2}$, with $f(0, \Delta p)=\frac{3}{2} \mu^{(r)}(\Delta p)^{2}$ and $f^{(r)} \rightarrow+\infty$ as $\left(\varepsilon_{e q}^{t r}\right)^{2} \rightarrow \infty$ (Fig. 1(a)). The function is formally extended on $\left(\varepsilon_{e q}^{t r}\right)^{2}<0$ as $\left.f^{(r)}\left(\varepsilon_{e q}^{t r}\right)^{2}, \Delta p\right) \equiv+\infty$. Therefore, $f^{(r)}$ is convex w.r.t. $\left(\varepsilon_{e q}^{t r}\right)^{2}$ (and lower semi-continuous).

In order to introduce linear comparison properties, and inspiring from the procedure of Ponte Castañeda (1991), the Legendre transform of $f^{(r)}$ is computed:

$$
f^{(r) *}\left(\frac{3}{2} \mu_{0}^{(r)}, \Delta p\right)=\sup _{\left(\varepsilon_{e q}^{t r}\right)^{2} \geq 0}\left\{\frac{3}{2} \mu_{0}^{(r)}\left(\varepsilon_{e q}^{t r}\right)^{2}-f^{(r)}\left(\left(\varepsilon_{e q}^{t r}\right)^{2}, \Delta p\right)\right\},
$$

where the non-negativeness of $\left(\varepsilon_{e q}^{t r}\right)^{2}$ is ensured by the definition of $f^{(r)}$ for negative $\left(\varepsilon_{e q}^{t r}\right)^{2}$. The expression between curly brackets in Eq. (56) is maximized by setting its derivative w.r.t. $\left(\varepsilon_{e q}^{t r}\right)^{2}$ equal to zero, which yields the following expression for the dual variable:

$$
\mu_{0}^{(r)}=\mu^{(r)}\left(1-\frac{\Delta p}{\varepsilon_{e q}^{t r}}\right)
$$

Solving for $\left(\varepsilon_{e q}^{\text {tr }}\right)^{2}$ gives

$$
\left(\varepsilon_{e q}^{t r}\right)^{2}=\left(\frac{\mu^{(r)}}{\mu^{(r)}-\mu_{0}^{(r)}}\right)^{2}(\Delta p)^{2} .
$$

An explicit expression of the dual convex of $f^{(r)}$ is obtained by substituting (58) into (56) (Fig. 1(b)):

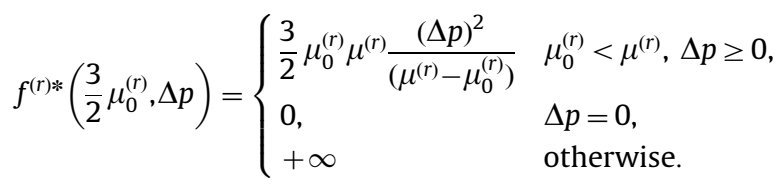

As $f^{(r)}$ is convex (and lower semi-continuous), the bidual $f^{(r) * * *} \equiv\left(f^{(r) * *}\right.$ of $f^{(r)}$ is $f^{(r)}$ itself:

$$
f^{(r)}\left(\left(\varepsilon_{e q}^{t r}\right)^{2}, \Delta p\right)=f^{(r) * * *}\left(\left(\varepsilon_{e q}^{t r}\right)^{2}, \Delta p\right)=\sup _{\mu_{0}^{(r)} \leq \mu^{(r)}}\left\{\frac{3}{2} \mu_{0}^{(r)}\left(\varepsilon_{e q}^{t r}\right)^{2}-f^{(r) *}\left(\frac{3}{2} \mu_{0}^{(r)}, \Delta p\right)\right\} .
$$

Expression (60) of $f^{(r)}$ is now introduced into expression (54) of the elastic free energy, leading to

$$
\Psi^{e(r)}\left(\boldsymbol{\varepsilon}^{t r}, \Delta p\right)=\sup _{\mu_{0}^{(r)} \leq \mu^{(r)}}\left\{W_{0}^{(r)}\left(\boldsymbol{\varepsilon}^{t r}, \mu_{0}^{(r)}\right)-f^{(r) *}\left(\frac{3}{2} \mu_{0}^{(r)}, \Delta p\right)\right\},
$$

a

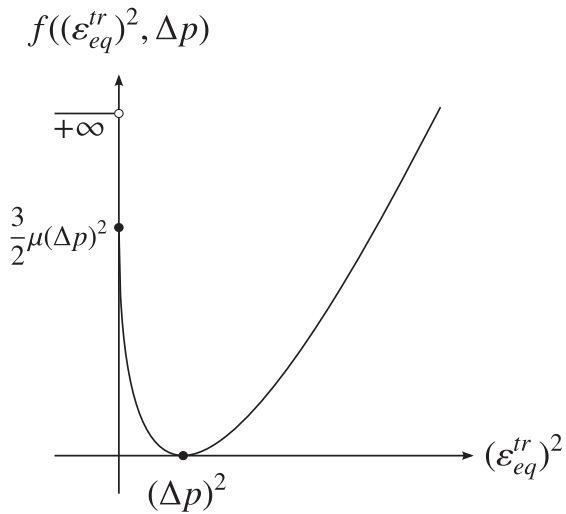

b

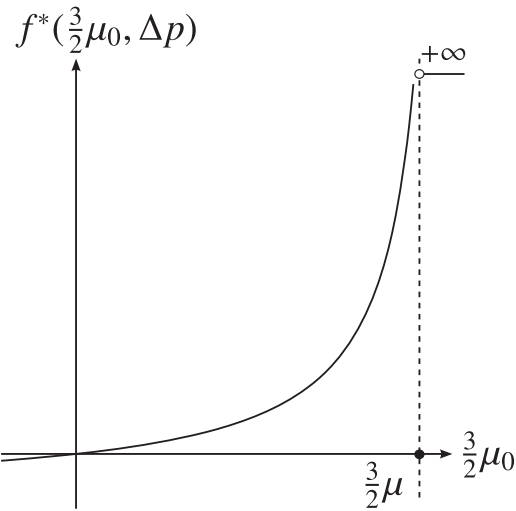

Fig. 1. The function $f\left(\left(\varepsilon_{e q}^{t r}\right)^{2}, \Delta p\right)$ (a) and its convex dual by the Legendre transform, $f^{*}\left(\frac{3}{2} \mu_{0}, \Delta p\right)$ (b). $\Delta p$ acts as a parameter, which is taken positive in the figures. 
where $W_{0}^{(r)}$ is similar in form to the elastic energy of a linear, isotropic material:

$$
W_{0}^{(r)}\left(\boldsymbol{\varepsilon}^{t r}, \mu_{0}^{(r)}\right)=\frac{9}{2} \kappa^{(r)}\left(\varepsilon_{m}^{t r}\right)^{2}+\frac{3}{2} \mu_{0}^{(r)}\left(\varepsilon_{e q}^{t r}\right)^{2}=\frac{1}{2} \varepsilon^{t r}: \boldsymbol{C}_{0}^{(r)}: \boldsymbol{\varepsilon}^{t r}=\frac{1}{2}\left(\boldsymbol{\varepsilon}-\boldsymbol{\varepsilon}_{n}^{p}\right): \boldsymbol{C}_{0}^{(r)}:\left(\boldsymbol{\varepsilon}-\boldsymbol{\varepsilon}_{n}^{p}\right),
$$

with $\boldsymbol{C}_{0}^{(r)}$ given by

$$
\boldsymbol{C}_{0}^{(r)}=3 \kappa^{(r)} \boldsymbol{I}^{\mathrm{Vol}}+2 \mu_{0}^{(r)} \boldsymbol{I}^{\mathrm{dev}} .
$$

Therefore, the dual variable $\mu_{0}^{(r)}$ introduced by the Legendre transform plays the role of a shear modulus in a fictitious linear elastic material with potential (62). Accordingly, it should be positive everywhere. It will be shown that it is indeed the case according to the model proposed in the next section. Note that $\mu_{0}^{(r)}$ fluctuates within phase $r$, according to its expression (57). On the other hand, the plastic strain $\varepsilon_{n}^{p}$ plays the role of an eigenstrain field applied to the linear comparison material.

The linearization suggested by the variational procedure can be interpreted as follows. Using expression (61) for the elastic part of the free energy, the local stress in the composite is given by

$$
\boldsymbol{\sigma}=\frac{\partial \Psi^{e(r)}}{\partial \boldsymbol{\varepsilon}^{t r}}: \frac{\partial \boldsymbol{\varepsilon}^{t r}}{\partial \boldsymbol{\varepsilon}^{e}}=\boldsymbol{C}_{0}^{(r)}: \boldsymbol{\varepsilon}^{t r}
$$

Thus, the comparison moduli $\boldsymbol{C}_{0}^{(r)}$ play the role of secant moduli in the stress-trial strain relation (Fig. 2). The linearization technique involved in the present approach can therefore be referred to as a trial, secant method.

Remark 3. Interestingly, the comparison shear modulus $\mu_{0}$ (57) coincides with coefficient $k_{2}$ in the following spectral decomposition of the algorithmic tangent operator of $\mathrm{J}_{2}$ plasticity:

$$
\boldsymbol{C}^{\text {alg }}=3 k_{1} \boldsymbol{C}^{(1)}+2 k_{2} \boldsymbol{C}^{(2)}+2 k_{3} \boldsymbol{C}^{(3)},
$$

where tensors $\boldsymbol{C}^{(i)}$ are given by

$$
\boldsymbol{C}^{(1)}=\boldsymbol{I}^{\mathrm{vol}}, \quad \boldsymbol{C}^{(3)}=\frac{2}{3} \boldsymbol{N} \otimes \boldsymbol{N}, \quad \boldsymbol{C}^{(2)}=\boldsymbol{I}^{\mathrm{dev}}-\frac{2}{3} \boldsymbol{N} \otimes \boldsymbol{N},
$$

and satisfy: $\boldsymbol{C}^{(i)}: \boldsymbol{C}^{(j)}=\delta_{i j} \boldsymbol{C}^{(i)}$ (no sum over $i$ ). The decomposition (65) was introduced by Ponte Castañeda (1996) for tangent operators in nonlinear elasticity. When applied to the algorithmic tangent operator of $\mathrm{J}_{2}$ elasto-plasticity, the coefficients $k_{i}$ read (Doghri and Ouaar, 2003, recall that $\sigma_{e q}^{t r}=3 \mu \varepsilon_{e q}^{t r}$ )

$$
k_{1}=\kappa, \quad k_{2}=\mu\left(1-3 \mu \frac{\Delta p}{\sigma_{e q}^{t r}}\right), \quad k_{3}=\mu\left(1-\frac{3 \mu}{3 \mu+R^{\prime}(p)}\right) .
$$

\subsection{Overall potential}

We now use the linearization technique presented in the previous section to express the overall potential of the composite in terms of the effective potential of a LCC. Substituting expression (61) together with (62) into that of the effective potential (50) leads to

$$
\bar{W}_{\Delta}(\overline{\boldsymbol{\varepsilon}})=\inf _{\varepsilon \in \mathcal{K}(\overline{\boldsymbol{\varepsilon}})}\left\langle\inf _{\Delta p \geq 0} \sup _{0<\mu_{0}^{(s)} \leq \mu^{(s)}}\left\{\sum_{r=1}^{n} \chi^{(r)}(\boldsymbol{x})\left(W_{0}^{(r)}\left(\boldsymbol{\varepsilon}-\varepsilon_{n}^{p}, \mu_{0}^{(r)}\right)-f^{(r) *}\left(\frac{3}{2} \mu_{0}^{(r)}, \Delta p\right)+\psi^{p(r)}\left(p_{n}+\Delta p\right)-\psi_{n}^{(r)}(\boldsymbol{x})+\Delta t \phi^{(r)}\left(\frac{\Delta p}{\Delta t}\right)\right)\right\}\right\rangle,
$$

in which the condition $\Delta p \geq 0$ follows from the specific form (41) of the dissipation function. The expression between curly brackets is convex in $\boldsymbol{\varepsilon}$ (under the ansatz that $\mu_{0}^{(r)}$ is positive) and concave w.r.t. $\mu_{0}^{(r)}$. According to the saddle-point theorem (Rockafellar, 1970), the order of the infimum w.r.t. $\varepsilon$ and supremum w.r.t. $\mu_{0}^{(r)}$ can be interchanged, and an alternative

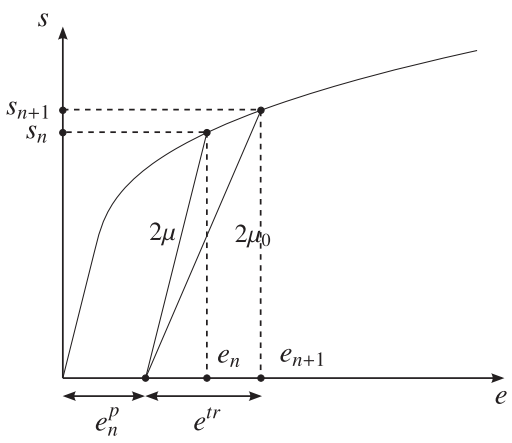

Fig. 2. The proposed variational formulation can be interpreted as a secant method based on the elastic trial strain: $\varepsilon_{n+1}^{t r}=\varepsilon_{n+1}-\varepsilon_{n}^{p}$. 
representation is obtained:

$$
\bar{W}_{\Delta}(\overline{\boldsymbol{\varepsilon}})=\inf _{\Delta p \geq 0} \sup _{0<\mu_{0}^{(s)} \leq \mu^{(s)}}\left\{\bar{W}_{0}\left(\overline{\boldsymbol{\varepsilon}}, \mu_{0}^{(s)}\right)+\left\langle\sum_{r=1}^{n} \chi^{(r)}(\boldsymbol{x})\left(-f^{(r) *}\left(\frac{3}{2} \mu_{0}^{(r)}, \Delta p\right)+\psi^{p(r)}\left(p_{n}+\Delta p\right)-\psi_{n}^{(r)}(\boldsymbol{x})+\Delta t \phi^{(r)}\left(\frac{\Delta p}{\Delta t}\right)\right)\right\rangle\right\},
$$

where $\bar{W}_{0}$ is the effective potential of a LCC characterized by phase potentials $W_{0}^{(r)}$ :

$$
\bar{W}_{0}\left(\overline{\boldsymbol{\varepsilon}}, \mu_{0}^{(s)}\right)=\inf _{\boldsymbol{\varepsilon} \in \mathcal{K}(\bar{\varepsilon})}\left\langle\sum_{r=1}^{n} \chi^{(r)}(\boldsymbol{x}) W_{0}^{(r)}\left(\boldsymbol{\varepsilon}-\boldsymbol{\varepsilon}_{n}^{p}, \mu_{0}^{(r)}\right)\right\rangle .
$$

The expression between curly brackets in (69) is not convex w.r.t. $\Delta p$, so that the order of the infimum and supremum operations may not be inverted. Accounting for the stationarity w.r.t. the fields $\mu_{0}^{(r)}$ and $\Delta p$, the overall stress of the composite is given by

$$
\overline{\boldsymbol{\sigma}}=\frac{\partial \bar{W}_{\Delta}}{\partial \overline{\boldsymbol{\varepsilon}}}(\overline{\boldsymbol{\varepsilon}})=\frac{\partial \bar{W}_{0}}{\partial \overline{\boldsymbol{\varepsilon}}}\left(\overline{\boldsymbol{\varepsilon}}, \mu_{0}^{(s)}\right) .
$$

The formulation (69) of the homogenization problem, supplemented by the local flow rule (21), is completely equivalent to the original one (50), and is therefore as complicated to solve. However, it involves the effective energy of a LCC, which constitutes the first step towards the derivation of estimates.

\subsection{Approximation by piecewise uniform shear moduli}

A straightforward (and probably unavoidable) approximation to formulation (69) consists in considering piecewise uniform shear moduli within the LCC:

$$
\mu_{0}(\boldsymbol{x})=\sum_{r=1}^{N} \chi^{(r)}(\boldsymbol{x}) \mu_{0}^{(r)},
$$

where $\mu_{0}^{(r)}$ is now uniform in phase $r$. This corresponds to a restriction of the solutions space in the variational problem (69). In (72), it is assumed that the spatial distribution of the phases in the LCC coincides with the one in the actual composite. ${ }^{2}$

Approximation (72) also implies piecewise uniformity of the optimal field $\Delta p(\boldsymbol{x})$, solution of the variational problem (69). Indeed, consider the composite in a strain- and stress-free configuration at $t=t_{0}$, so that $p_{n}$ is initially piecewise uniform (and zero). As all terms involving $p$ and/or $\Delta p$ in the functional between curly brackets in Eq. (69) are piecewise uniform, it follows that $\Delta p(\boldsymbol{x})$ solution of the infimum problem at $t_{1}$ is also piecewise uniform. Applying the same reasoning at each subsequent time step, the fields $\Delta p(\boldsymbol{x})$, as well as $p(\boldsymbol{x})$ at any time $t_{n+1}$ are necessarily piecewise uniform.

\section{An estimate based on uniform eigenstrain}

The piecewise uniformity of the shear moduli and the internal variable $p$ (as a consequence) dramatically reduces the difficulty of problem (69). However, the eigenstrain field $\varepsilon_{n}^{p}$ in (70) fluctuates within each plastic phase, preventing a direct application of linear estimates for thermoelastic composites. Therefore, an additional approximation is required, which might consist in considering a uniform, reference plastic strain at $t_{n}$ for each phase in expression (70). A rather intuitive choice is to set the reference plastic strain equal to the average of the plastic strain in the phase. Unfortunately, results obtained so far under this assumption turned out to be inconsistent in most examples of particulate composites reinforced by elastic inclusions (Brassart, 2011).

The estimate proposed and validated in the sequel is based on a different, and yet simpler modeling assumption of a uniform reference plastic strain for the whole composite. The effective potential (70) of the LCC is then approximated as

$$
\bar{W}_{0}\left(\overline{\boldsymbol{\varepsilon}}, \mu_{0}^{(s)}\right) \approx \tilde{W}_{0}\left(\overline{\boldsymbol{\varepsilon}}, \mu_{0}^{(s)}\right)=\inf _{\boldsymbol{\varepsilon} \in \mathcal{K}(\overline{\boldsymbol{\varepsilon}})}\left\langle\sum_{r=1}^{n} \chi^{(r)}(\boldsymbol{x}) W_{0}^{(r)}\left(\boldsymbol{\varepsilon}-\hat{\boldsymbol{\varepsilon}}_{n}^{p}, \mu_{0}^{(r)}\right)\right\rangle,
$$

where $\hat{\boldsymbol{\varepsilon}}_{n}^{p}$ is the reference plastic strain, given by

$$
\hat{\boldsymbol{\varepsilon}}_{n}^{p} \equiv\left\langle\boldsymbol{\varepsilon}_{n}^{p}\right\rangle \text {. }
$$

Then, the effective potential of the LCC is simply given by

$$
\tilde{W}_{0}\left(\overline{\boldsymbol{\varepsilon}}, \mu_{0}^{(s)}\right)=\frac{1}{2}\left(\overline{\boldsymbol{\varepsilon}}-\left\langle\boldsymbol{\varepsilon}_{n}^{p}\right\rangle\right): \overline{\boldsymbol{C}}_{0}:\left(\overline{\boldsymbol{\varepsilon}}-\left\langle\boldsymbol{\varepsilon}_{n}^{p}\right\rangle\right),
$$

where $\overline{\boldsymbol{C}}_{0}$ is the overall elastic stiffness of the LCC, to be computed from any linear scheme suited for the microstructure under consideration.

\footnotetext{
${ }^{2}$ This prescription is not absolutely necessary, nor necessarily optimal, as noted by Suquet (1993). However, the question of considering a LCC with a microstructure different from the actual one is not investigated in the present work.
} 
The proposed simplified model considers the LCC to be subjected to a uniform pre-deformation corresponding to the average plastic deformation at the previous time step. With such simplification, inter-phase (as well as intra-phase) plastic strain heterogeneities are overlooked when solving the LCC problem. Note however that the actual plastic strain field is not supposed to be uniform: the uniform reference plastic strain is used for the localization step only. Updates for the perphase averages of the plastic strain will be described later. Despite the apparent crudeness of prescription (74), valuable predictions are obtained when the model is applied to two-phase particulate composites, as shown in Section 6.

Remark 4. Adopting the following change of variable: $\boldsymbol{\varepsilon}^{\prime}(\boldsymbol{x}) \equiv \boldsymbol{\varepsilon}(\boldsymbol{x})-\hat{\boldsymbol{\varepsilon}}_{n}^{p}$, where $\boldsymbol{\varepsilon}(\boldsymbol{x})$ is the strain field solution of problem (73), the latter is equivalently reexpressed as

$$
\tilde{W}_{0}\left(\overline{\boldsymbol{\varepsilon}}, \mu_{0}^{(s)}\right)=\inf _{\boldsymbol{\varepsilon}^{\prime} \in \mathcal{K}\left(\overline{\boldsymbol{\varepsilon}}^{t^{r}}\right)}\left\langle\sum_{r=1}^{N} \chi^{(r)}(\boldsymbol{x}) W_{0}^{(r)}\left(\boldsymbol{\varepsilon}^{\prime}, \mu_{0}^{(r)}\right)\right\rangle,
$$

where $\overline{\boldsymbol{\varepsilon}}^{t r} \equiv \overline{\boldsymbol{\varepsilon}}-\left\langle\boldsymbol{\varepsilon}_{n}^{p}\right\rangle$. Note that the field $\boldsymbol{\varepsilon}^{\prime}$ is compatible.

According to (71), the macroscopic stress in the nonlinear composite is given by the macroscopic stress in the LCC:

$$
\overline{\boldsymbol{\sigma}}=\overline{\boldsymbol{C}}_{0}:\left(\overline{\boldsymbol{\varepsilon}}-\left\langle\boldsymbol{\varepsilon}_{n}^{p}\right\rangle\right)
$$

Relation (77) also implies that the volume average of the stress in the nonlinear composite and in the LCC coincide. Therefore, it seems natural to approximate per-phase averages of the stress in the nonlinear composite by corresponding ones in the LCC:

$$
\langle\boldsymbol{\sigma}\rangle_{r}=\left\langle\boldsymbol{\sigma}^{\prime}\right\rangle_{r},
$$

where $\boldsymbol{\sigma}^{\prime}$ denotes the stress field in the LCC with uniform eigenstrain. We will further assume that moments of the trial strain field in the nonlinear composite are approximated by corresponding moments of the field $\boldsymbol{\varepsilon}^{\prime}$ computed in the LCC:

$$
\left\langle\boldsymbol{\varepsilon}^{\text {tr }}\right\rangle_{r}=\left\langle\boldsymbol{\varepsilon}^{\prime}\right\rangle_{r}, \quad\left\langle\left(\varepsilon_{e q}^{\text {tr }}\right)^{2}\right\rangle_{r}=\left\langle\left(\varepsilon_{e q}^{\prime}\right)^{2}\right\rangle_{r}
$$

\subsection{Optimization w.r.t. $\mu_{0}^{(r)}$ and $\Delta p^{(r)}$}

Adopting expression (76) for the effective potential of the LCC, we now address the optimization w.r.t. $\mu_{0}^{(r)}$ and $\Delta p^{(r)}$ in (69). The stationarity condition w.r.t. $\mu_{0}^{(r)}$ writes

$$
\frac{\partial \tilde{W}_{0}}{\partial \mu_{0}^{(r)}}-c_{r}\left\langle\frac{\partial f_{*}^{(r)}}{\partial \mu_{0}^{(r)}}\left(\mu_{0}^{(r)}, \Delta p^{(r)}\right)\right\rangle_{r}=0
$$

A classical result in the homogenization of linear composites indicates that the first term in the left-hand side member can be rewritten in terms of the second moment of the strain field in the LCC as (Bobeth and Diener, 1986; Kreher, 1990; Ponte Castañeda and Suquet, 1998)

$$
\frac{\partial \tilde{W}_{0}}{\partial \mu_{0}^{(r)}}=\frac{3}{2} c_{r}\left\langle\left(\varepsilon_{e q}^{\prime}\right)^{2}\right\rangle_{r}=\frac{3}{2} c_{r}\left\langle\left(\varepsilon_{e q}^{t r}\right)^{2}\right\rangle_{r}
$$

where the last equality follows from assumption (79). On the other hand, the derivative of $f_{*}^{(r)}$ w.r.t. $\mu_{0}^{(r)}$ gives

$$
\left.\frac{\partial f_{*}^{(r)}}{\partial \mu_{0}^{(r)}}\left(\mu_{0}^{(r)}, \Delta p^{(r)}\right)=\frac{3}{2} \frac{\mu^{(r)} \Delta p^{(r)}}{\mu^{(r)}-\mu_{0}^{(r)}}\right)^{2}
$$

Combining the last three equations and accounting for the homogeneity of $\Delta p^{(r)}$, one obtains an explicit expression of the effective shear moduli which is similar in form to (57):

$$
\mu_{0}^{(r)}=\mu^{(r)}\left(1-\frac{\Delta p^{(r)}}{\sqrt{\left\langle\left(\varepsilon_{e q}^{t r}\right)^{2}\right\rangle_{r}}}\right) .
$$

Taking the stationarity w.r.t. $\mu_{0}^{(r)}$ into account, the minimization w.r.t. $\Delta p^{(r)}$ yields the following condition in phase $r$ :

$$
-3 \mu^{(r)} \frac{\mu_{0}^{(r)}}{\mu^{(r)}-\mu_{0}^{(r)}} \Delta p^{(r)}+R^{(r)}\left(p_{n}^{(r)}+\Delta p^{(r)}\right)+\frac{\partial \phi^{(r)}}{\partial \dot{p}}\left(\frac{\Delta p^{(r)}}{\Delta t}\right)=0,
$$

or, making use of (83):

$$
-3 \mu^{(r)} \sqrt{\left\langle\left(\varepsilon_{e q}^{t r}\right)^{2}\right\rangle_{r}}+3 \mu^{(r)} \Delta p^{(r)}+R^{(r)}\left(p_{n}^{(r)}+\Delta p^{(r)}\right)+\frac{\partial \phi^{(r)}}{\partial \dot{p}}\left(\frac{\Delta p^{(r)}}{\Delta t}\right)=0 .
$$

Recalling Eq. (33), Eq. (85) can be interpreted as a homogenized radial return equation for phase $r$. A positive $\Delta p^{(r)}$ solution of (85) is found if the slope of the functional to minimize, computed for $\Delta p^{(r)} \rightarrow 0$, is negative. The slope actually is the 
left-hand side of (85). The unique yield criterion for phase $r$ reads

$$
-3 \mu^{(r)} \sqrt{\left\langle\left(\varepsilon_{e q}^{t r}\right)^{2}\right\rangle_{r}}+R^{(r)}\left(p_{n}^{(r)}\right)+\sigma_{Y}^{(r)}<0 .
$$

In the yield criterion (86) the second moment $\left\langle\left(\varepsilon_{e q}^{t r}\right)^{2}\right\rangle_{r}$ is computed on the LCC characterized by the elastic shear moduli $\mu^{(r)}$. Indeed, $\mu_{0}^{(r)} \rightarrow \mu^{(r)}$ as $\Delta p^{(r)} \rightarrow 0$, according to expression (83). If the yield criterion (86) is not satisfied, the increment is elastic in phase $r$, and the minimum is achieved for $\Delta p^{(r)}=0$. Otherwise, the radial return condition (85) takes the familiar form (35):

$$
-3 \mu^{(r)} \sqrt{\left\langle\left(\varepsilon_{e q}^{t r}\right)^{2}\right\rangle_{r}}+3 \mu^{(r)} \Delta p^{(r)}+R^{(r)}\left(p_{n}^{(r)}+\Delta p^{(r)}\right)+\sigma_{Y}^{(r)}=0 .
$$

Since Eq. (87) may be rewritten as

$$
\sqrt{\left\langle\left(\varepsilon_{e q}^{t r}\right)^{2}\right\rangle_{r}}=\Delta p^{(r)}+\frac{1}{3 \mu^{(r)}}\left(R^{(r)}\left(p_{n}^{(r)}+\Delta p^{(r)}\right)+\sigma_{Y}^{(r)}\right),
$$

the second term of the right hand side is always positive. Hence, $\left.\sqrt{\left\langle\left(\varepsilon_{e q}^{t r}\right)^{2}\right\rangle_{r}}\right\rangle \Delta p^{(r)}$ and $\mu_{0}^{(r)}$ is always positive, as announced.

\subsection{Homogenized flow rule}

The proposed estimate of the composite mechanical response also requires computation of the phase average of the plastic strain (or plastic strain increment) at each time step:

$$
\left\langle\Delta \boldsymbol{\varepsilon}^{p}\right\rangle=\sum_{r=1}^{N} c_{r}\left\langle\Delta \boldsymbol{\varepsilon}^{p}\right\rangle_{r}=\sum_{r=1}^{N} c_{r} \Delta p^{(r)}\left\langle\frac{\boldsymbol{e}^{t r}}{\varepsilon_{e q}^{t r}}\right\rangle_{r} .
$$

The average plastic strain update is computed by making use of the following observations. On the one hand, the phase average of the stress is given by

$$
\langle\boldsymbol{\sigma}\rangle_{r}=\boldsymbol{C}^{e(r)}:\left\langle\boldsymbol{\varepsilon}^{t r}-\Delta \boldsymbol{\varepsilon}^{p}\right\rangle_{r}=\boldsymbol{C}^{e(r)}:\left\langle\boldsymbol{\varepsilon}^{t r}\right\rangle_{r}-2 \mu^{(r)}\left\langle\Delta \boldsymbol{\varepsilon}^{p}\right\rangle_{r} .
$$

On the other hand, according to (78), we also have

$$
\langle\boldsymbol{\sigma}\rangle_{r}=\left\langle\boldsymbol{\sigma}^{\prime}\right\rangle_{r}=\boldsymbol{C}_{0}^{(r)}:\left\langle\boldsymbol{\varepsilon}^{\prime}\right\rangle_{r}=\boldsymbol{C}^{e(r)}:\left\langle\boldsymbol{\varepsilon}^{\prime}\right\rangle_{r}-2 \mu^{(r)} \Delta p^{(r)} \frac{\left\langle\boldsymbol{e}^{\prime}\right\rangle_{r}}{\sqrt{\left\langle\left(\varepsilon_{e q}^{\prime}\right)^{2}\right\rangle_{r}}} .
$$

The last equality is obtained using expression (83) of the shear modulus $\mu_{0}^{(r)}$. Making use of assumption (79), a direct comparison of the last two expressions yields

$$
\left\langle\Delta \boldsymbol{\varepsilon}^{p}\right\rangle_{r}=\Delta p^{(r)}\left\langle\frac{\boldsymbol{e}^{t r}}{\varepsilon_{e q}^{t r}}\right\rangle_{r}=\Delta p^{(r)} \frac{\left\langle\boldsymbol{e}^{t r}\right\rangle_{r}}{\sqrt{\left\langle\left(\varepsilon_{e q}^{t r}\right)^{2}\right\rangle_{r}}},
$$

which defines an effective flow direction $\boldsymbol{N}^{(r)}$ for phase $r$ :

$$
\mathbf{N}^{(r)} \equiv \frac{\left\langle\boldsymbol{e}^{t r}\right\rangle_{r}}{\sqrt{\left\langle\left(\varepsilon_{e q}^{t r}\right)^{2}\right\rangle_{r}}} .
$$

Eq. (92) can be viewed as a homogenized plastic flow rule for the average plastic strain.

Remark 5. In general, $\boldsymbol{N}^{(r)}: \boldsymbol{N}^{(r)} \neq 3 / 2$. Instead:

$$
\boldsymbol{N}^{(r)}: \boldsymbol{N}^{(r)}=\frac{3}{2} \frac{\left(\left\langle\varepsilon^{t r}\right\rangle_{r}\right)_{e q}^{2}}{\left\langle\left(\varepsilon_{e q}^{t r}\right)^{2}\right\rangle_{r}},
$$

where the second-order moment of $\boldsymbol{\varepsilon}^{t r}$ is necessarily greater than the first-order moment, except in very specific situations where fields are homogeneous in each phase.

\subsection{Summary of the homogenization procedure}

Taking advantage of formulation (76) for the effective behavior of the LCC, algorithmic implementation is rather straightforward. We consider a composite in a strain- and stress-free configuration at $t=t_{0}$. On a time interval $\left[t_{n}, t_{n+1}\right]$, history variables at $t_{n}$ are given for each phase $r:\left\langle\varepsilon_{n}^{p}\right\rangle_{r}$ and $\left\langle p_{n}\right\rangle_{r}$. Given $\overline{\boldsymbol{\varepsilon}}$, the macroscopic strain at $t_{n+1}$, the problem is to compute the macroscopic stress $\overline{\boldsymbol{\sigma}}$. In the proposed numerical procedure, we iterate on the value of the reference shear moduli $\mu_{0}^{(r)}$.

- Elastic predictor step. Taking $\mu_{0}^{(r)}=\mu^{(r)}$ :

1. Compute the effective stiffness $\overline{\boldsymbol{C}}_{0}$ according to the chosen homogenization scheme for a linear elastic composite. Next, compute $\left\langle\left(\varepsilon_{e q}^{t r}\right)^{2}\right\rangle_{r}$ according to Eq. (81). 
2. Evaluate the yield criterion (86) in each phase:

$$
k^{(r)}(r) \equiv-3 \mu^{(r)} \sqrt{\left\langle\left(\varepsilon_{e q}^{t r}\right)^{2}\right\rangle_{r}}+R^{(r)}\left(p_{n}^{(r)}\right)+\sigma_{Y}^{(r)} .
$$

3. If $k^{(r)} \geq 0$ : the increment is elastic in phase $r$, and $\mu_{0}^{(r)}=\mu^{(r)}$ and $\Delta p^{(r)}=0$.

4. Otherwise, $\mu_{0}^{(r)}$ is smaller than $\mu^{(r)}$ and it must be found iteratively.

- Plastic correction step. Iteration (i) (upper index (i) omitted for simplicity). Compute the effective stiffness $\overline{\boldsymbol{C}}_{0}$ according to the chosen homogenization scheme for a linear elastic composite. For each phase $r$ in which plastic yielding occurs:

1. Compute $\left\langle\left(\varepsilon_{e q}^{\text {tr }}\right)^{2}\right\rangle_{r}$ according to Eq. (81).

2. Compute $\Delta p^{(r)}$ according to Eq. (83):

$$
\Delta p^{(r)}=\frac{\mu^{(r)}-\mu_{0}^{(r)}}{\mu^{(r)}} \sqrt{\left\langle\left(\varepsilon_{e q}^{t r}\right)^{2}\right\rangle_{r}}
$$

3. Compute the residual (radial return Eq. (87)):

$$
F^{(r)} \equiv-3 \mu^{(r)} \sqrt{\left\langle\left(\varepsilon_{e q}^{t r}\right)^{2}\right\rangle_{r}}+3 \mu^{(r)} \Delta p^{(r)}+R^{(r)}\left(p_{n}^{(r)}+\Delta p^{(r)}\right)+\sigma_{Y}^{(r)} .
$$

Iterate on $\mu_{0}^{(r)}$ until the absolute value of the residual in each phase becomes lower than a given tolerance.

- After convergence. Compute the increment of average plastic strain:

$$
\left\langle\Delta \boldsymbol{\varepsilon}^{p}\right\rangle_{r}=\Delta p^{(r)} \frac{\left\langle\boldsymbol{e}^{t r}\right\rangle_{r}}{\sqrt{\left\langle\left(\varepsilon_{e q}^{t r}\right)^{2}\right\rangle_{r}}},
$$

and update the internal variables:

$$
\begin{aligned}
& \left\langle\boldsymbol{\varepsilon}^{p}\right\rangle_{r}=\left\langle\boldsymbol{\varepsilon}_{n}^{p}\right\rangle_{r}+\left\langle\Delta \boldsymbol{\varepsilon}^{p}\right\rangle_{r}, \\
& p^{(r)}=p_{n}^{(r)}+\Delta p^{(r)} .
\end{aligned}
$$

Finally, the macroscopic stress is obtained from the effective stiffness of the LCC as

$$
\overline{\boldsymbol{\sigma}}=\overline{\boldsymbol{C}}_{0}:\left(\overline{\boldsymbol{\varepsilon}}-\left\langle\varepsilon_{n}^{p}\right\rangle\right)=\langle\boldsymbol{\sigma}\rangle .
$$

In cases where an expression for the effective stiffness $\overline{\boldsymbol{C}}_{0}$ is available in closed form, the procedure is implemented using the Newton-Raphson method. In the examples of the next section, three iterations are typically sufficient to reach convergence.

\section{Application to two particle-reinforced composites}

In this section we compare the predictions of the simplified model proposed in Section 5 to reference results obtained from full-field finite element (FE) simulations for several two-phase composites. We consider composites made of a square array of spherical inclusions, which are frequently approximated by axisymmetric unit cells (Fig. 3(a)) allowing full-field computations at low cost. The geometry is meshed using the GMSH software (Geuzaine and Remacle, 2009), and a typical mesh comprises approximately 1000 elements and 2700 nodes (Fig. 3(b)). A convergence study was successfully conducted by comparing the predictions to those obtained with finer meshes (about 2500 elements). FE computations are performed using ABAQUS 6.9 (2009) using quadratic CAX6 and CAX8 elements. Reference, FE predictions are labeled "FE" in the figures.

Regarding the simplified model, two approaches have been pursued to solve the localization problem over the LCC. On the one hand, the LCC is homogenized "exactly" using the FE method. In this case, first- and second-order moments of stress and strain fields involved in the procedure are computed from direct volume averaging of the local fields in the LCC.
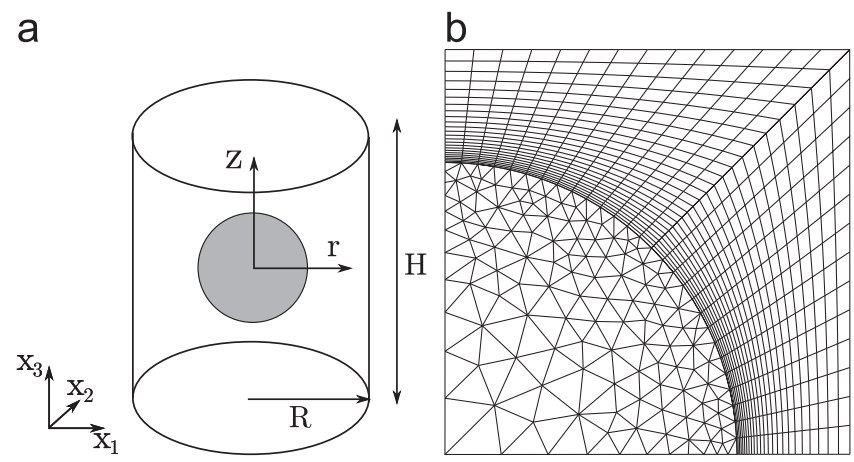

Fig. 3. (a) Reference predictions for composites with periodic microstructure are obtained considering cylindrical unit cells. (b) FE computations are performed using axisymmetric elements. 
In this way, the linearization procedure, together with the other approximation introduced in Section 5, are assessed while avoiding additional errors related to the use of an approximate linear homogenization scheme. This methodology was already used in order to evaluate the capabilities of linearization methods by Rekik et al. (2007) and Lahellec and Suquet (2007a). Corresponding results are labeled "VAR+FE" in the figures.

Alternatively, (hopefully) reliable estimates of the effective response of inclusion-reinforced linear elastic composites are provided by Hashin-Shtrikman (HS) lower bound (Hashin and Shtrikman, 1963; Willis, 1977). An advantage of the HS bounds is the relative simplicity of implementation: an expression of the effective stiffness of the LCC is then available in closed-form. The predictions of the variational method combined with the HS lower bound are labeled "VAR+HS-" in the figures.

The composites are subjected to uniaxial tension in the $z$-direction. The boundary conditions applied to the unit cell are the following:

$$
\begin{aligned}
& u_{3}(r, z=H / 2)=\bar{u}_{3}, \quad 0<r<R, \\
& u_{3}(r, z=0)=0, \quad 0<r<R, \\
& u_{1}(r=0, z)=0, \quad 0<z<H / 2, \\
& u_{1}(r=R, z)=\bar{u}_{1}, \quad 0<z<H / 2,
\end{aligned}
$$

with $H=2 R$. The displacement $\bar{u}_{3}$ is prescribed, while $\bar{u}_{1}$ is a priori unknown as the $r=R$ boundary is traction-free. Loading involves 50 time increments unless otherwise indicated.

\subsection{Elastic inclusions, elasto-plastic matrix}

The material under consideration is a metal matrix composite (MMC) with the following properties:

- Inclusions (phase 1): $E=400 \mathrm{GPa}, v=0.2$.

- Matrix (phase 2): $E=75 \mathrm{GPa}, v=0.3, \sigma_{Y}=75 \mathrm{MPa}, R(p)=h p^{n}, h=400 \mathrm{MPa}, n=0.4$ or $n=0.05$.

Two volume fractions of inclusions are considered: $c_{1}=0.15$ and $c_{1}=0.30$. MMC's with similar material properties were previously considered by several authors aiming to assess homogenization models (Segurado et al., 2002; Michel and Suquet, 2003; Doghri and Ouaar, 2003; González et al., 2004; Chaboche et al., 2005; Pierard et al., 2007) so that the predictive capabilities of the present approach can easily be evaluated w.r.t. those schemes.

The effective response for both hardening exponents and volume fractions is presented in Fig. 4. The VAR+FE model gives satisfying predictions for both volume fractions in the case $n=0.4$, while it overestimates the reference response when $n=0.05$. In the latter case, better predictions are obtained using HS lower bound to homogenize the LCC (VAR+HSmodel), due to the compensation of errors between the underestimation brought by the HS lower bound and the overestimation due to the present choice of LCC. On the other hand, the prediction of the VAR+HS-model are too soft in the case $c_{1}=0.30$ and $n=0.4$.

The accuracy of the model regarding the phase response is assessed in Fig. 5 taking $c_{1}=0.15$. For both hardening exponents, the proposed estimate correctly predicts the matrix response, even without the additional underestimation brought by the HS model. The evolution of the accumulated plastic strain in the matrix is also very well captured by the homogenization models (not shown). The method is less accurate regarding the inclusion response. There is a large discrepancy between VAR+FE and VAR+HS-results observed in the inclusions, while they are remarkably close in the matrix.

Previous examples showed that the model is less accurate when the matrix presents weak hardening. A limit case is obtained considering a perfectly plastic matrix: $R(p)=0$ (other material properties left unchanged). As expected from previous observations, the VAR+FE model overestimates the effective response (Fig. 6(a)), due to an unsuccessful prediction of the inclusion response (Fig. 6(b)). Predictions are more accurate using HS lower bound to homogenize the LCC.

Convergence of the model is analyzed in Fig. 7 for $c_{1}=0.15$ and $n=0.4$, by successively considering 20,50 and 100 increments to achieve the total elongation. It has been checked against results obtained using a very large number of increments (up to 10000 ) that the curve with 100 increments can be considered as a converged result. The scatter between the stress-strain curves presented in the figure is very low, as required. Obviously, sufficiently small loading steps are needed to capture the elastic-plastic transition accurately. Similar conclusions hold for $n=0.05$ and the case of a perfectly plastic matrix.

The variational procedure is able to simulate non-monotonic loadings. Examples of uniaxial tension/compression tests for $c_{1}=0.15$ and $c_{1}=0.30$ are presented in Fig. 8, taking $n=0.4$. The figure shows comparable accuracy of the proposed models in tension and compression. For $c_{1}=0.30$, the full-field simulation demonstrates a Baushinger effect (early and progressive plastification in the unloading branches of the cycle). Such effect is related to the heterogeneity of the plastic strain field which developed during the initial step of uniaxial tension. The proposed model predicts a sharp elastic-plastic 

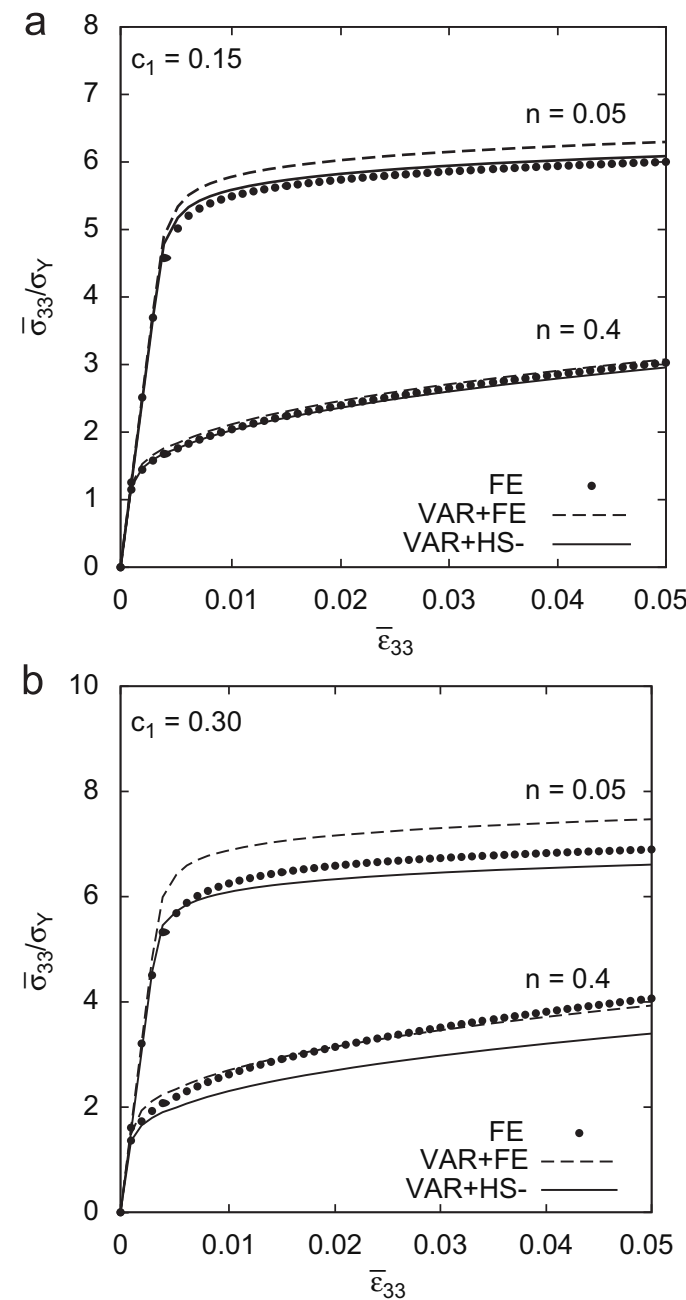

Fig. 4. Macroscopic response of a periodic composite with an elasto-plastic matrix and elastic inclusions for two volume fractions of inclusions: $c_{1}=0.15$ (a) and $c_{1}=0.30$ (b) and two different matrix hardening exponents: $n=0.05$ and $n=0.4$.
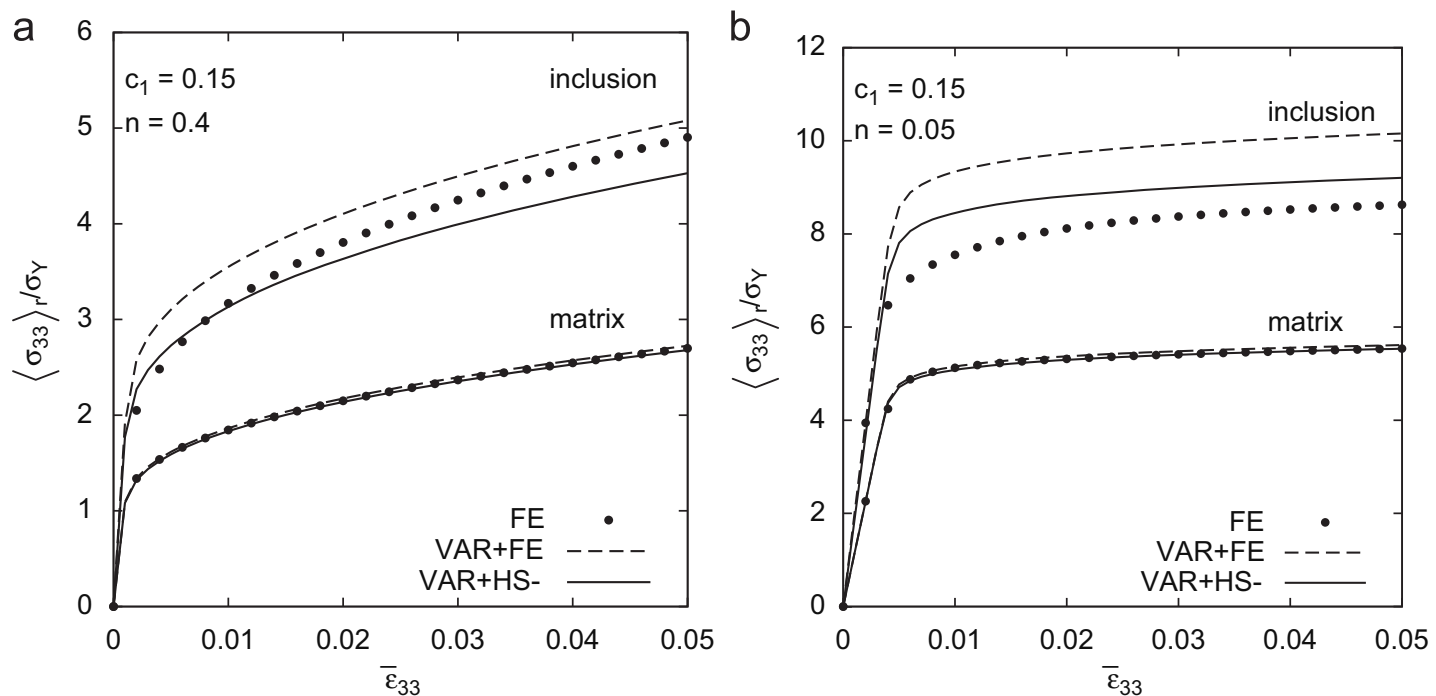

Fig. 5. Phase response of a periodic composite with an elasto-plastic matrix and elastic inclusions $\left(c_{1}=0.15\right)$ for two different matrix hardening exponents: $n=0.4$ (a) and $n=0.05$ (b). 

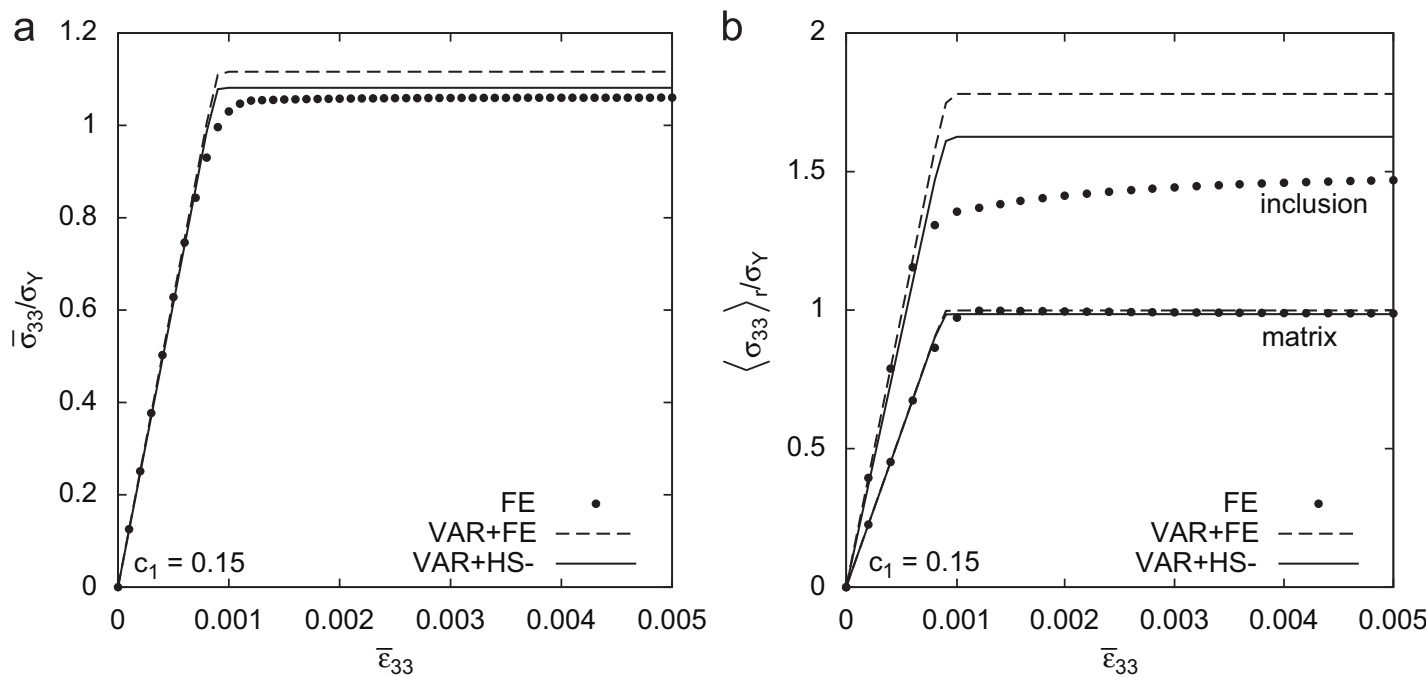

Fig. 6. Macroscopic (a) and phase (b) response of a periodic composite with a perfectly plastic matrix $(R(p)=0)$ and elastic inclusions $\left(c_{1}=0.15\right)$.

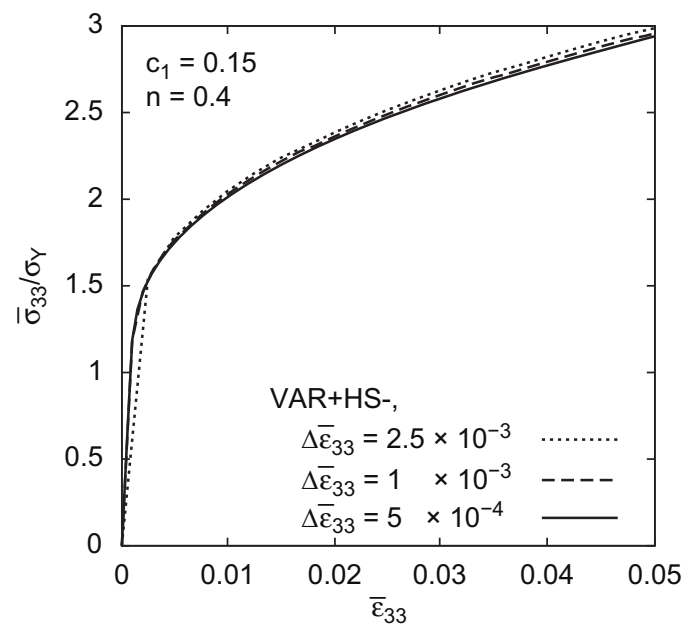

Fig. 7. Macroscopic response of a periodic composite with an elasto-plastic matrix $(n=0.4)$ and elastic inclusions $\left(c_{1}=0.15\right)$ as predicted by the VAR + HS-model, successively taking 20,50 and 100 strain increments to reach the final elongation.

transition in compression, as the homogenized yield criterion (86) for the matrix phase accounts for isotropic hardening only. Nevertheless, the stress level after the effective elastic-plastic transition is correctly predicted by the VAR+FE model.

\subsection{Elasto-plastic inclusions, elasto-plastic matrix}

We continue with a composite made of two elasto-plastic phases:

- Inclusions (phase 1): $E=400 \mathrm{GPa}, v=0.2, \sigma_{Y}=75 \mathrm{MPa}, R^{(1)}(p)=h^{(1)} p^{n^{(1)}}, h^{(1)}=1 \mathrm{GPa}, n^{(1)}=0.4$ or $n^{(1)}=0.05$.

- Matrix (phase 2): $E=75 \mathrm{GPa}, v=0.3, \sigma_{Y}=75 \mathrm{MPa}, R^{(2)}(p)=h^{(2)} p^{n^{(2)}}, h^{(2)}=400 \mathrm{MPa}, n^{(2)}=0.4$ or $n^{(2)}=0.05$.

The volume fraction of inclusions is $c_{1}=0.15$.

The effective response of the composite is presented in Fig. 9 for all combinations of inclusion and matrix hardening exponents. The responses for $n^{(1)}=0.05$ in Fig. 9(a) are very close to those presented in Fig. 4(a) for composites reinforced by elastic inclusions. Indeed, it can be checked that plastic deformations in the inclusions are negligible (although nonzero). On the contrary, when $n^{(1)}=0.4$ (Fig. 9(b)), plastic deformations are important in both phases. Interestingly, the $\mathrm{VAR}+\mathrm{FE}$ and VAR+HS-models predict almost identical effective response, whereas this is not necessarily true at the phase level. 

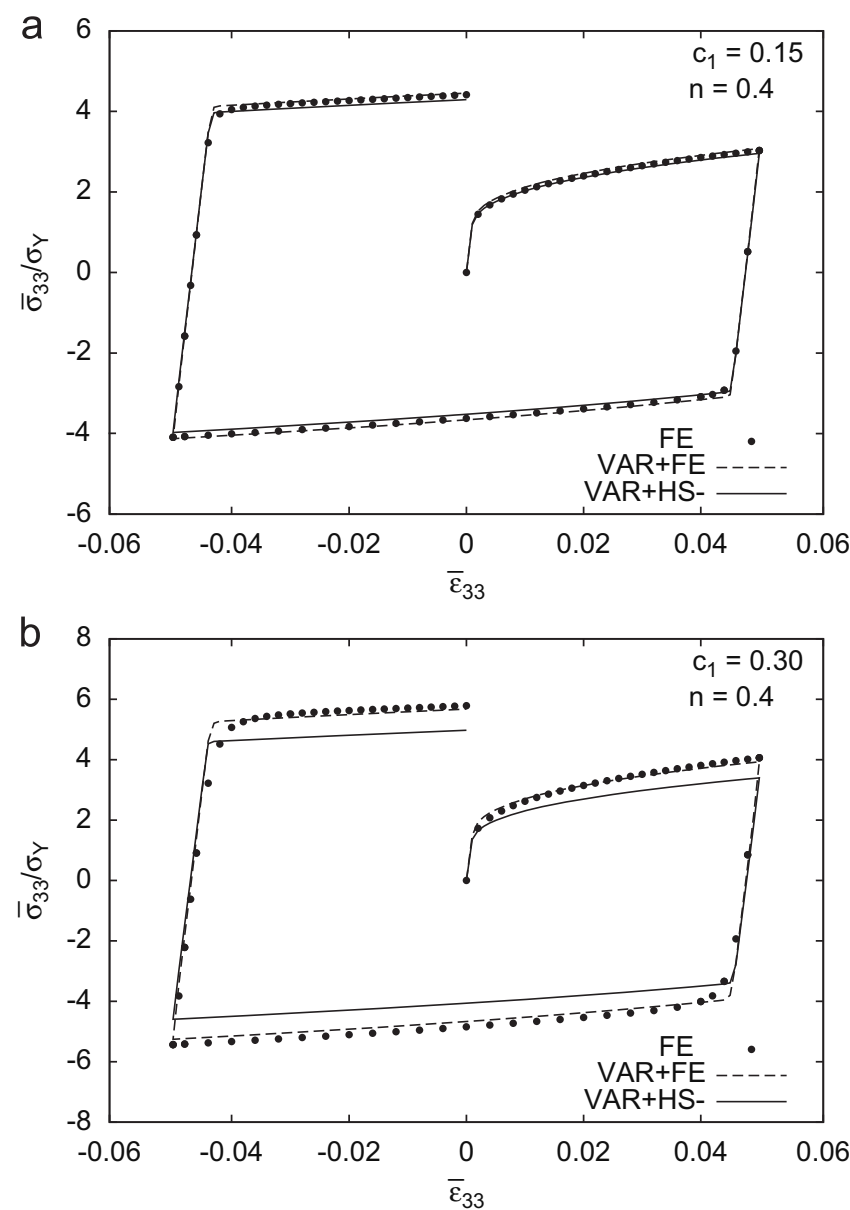

Fig. 8. Macroscopic response of a periodic composite with an elasto-plastic matrix $(n=0.4)$ and elastic inclusions, for $c_{1}=0.15$ (a) and $c_{1}=0.30(b)$.
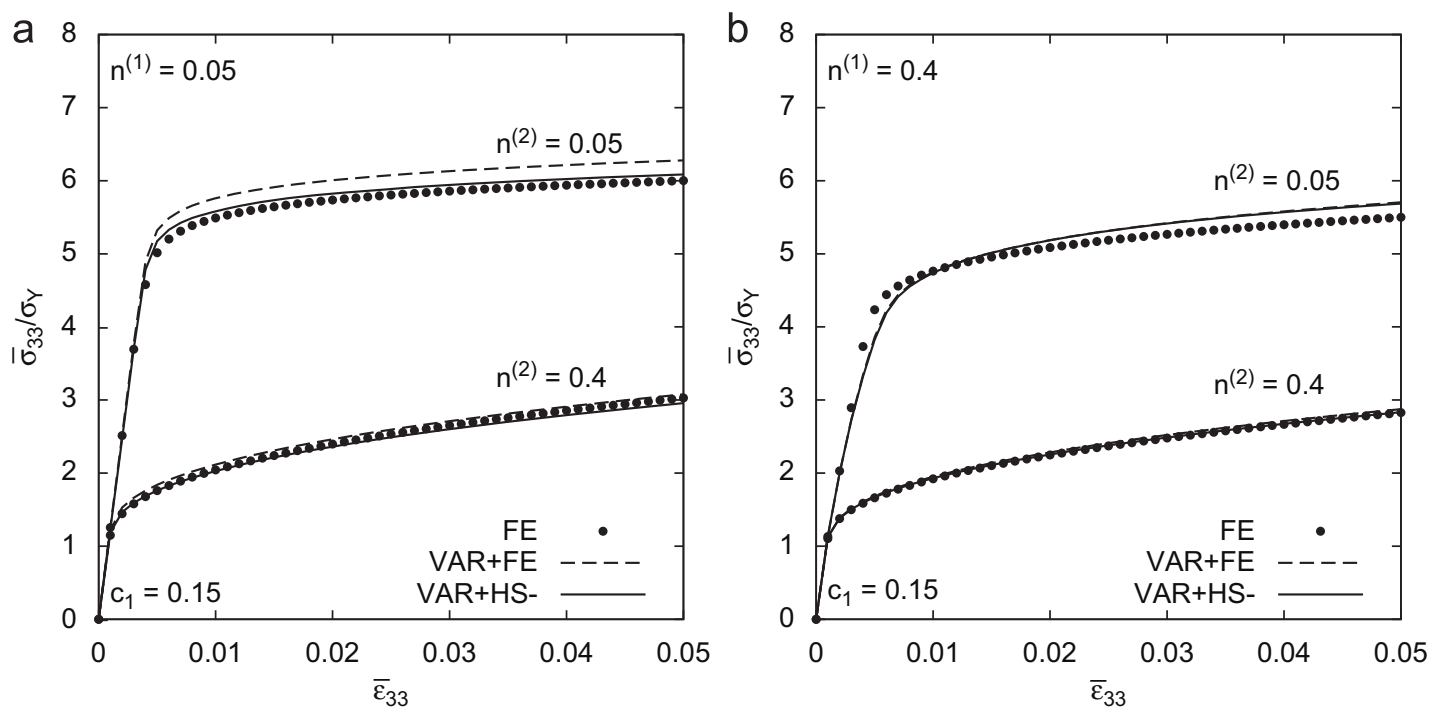

Fig. 9. Macroscopic response of a periodic composite with an elasto-plastic matrix and elasto-plastic inclusions having different strain hardening. 

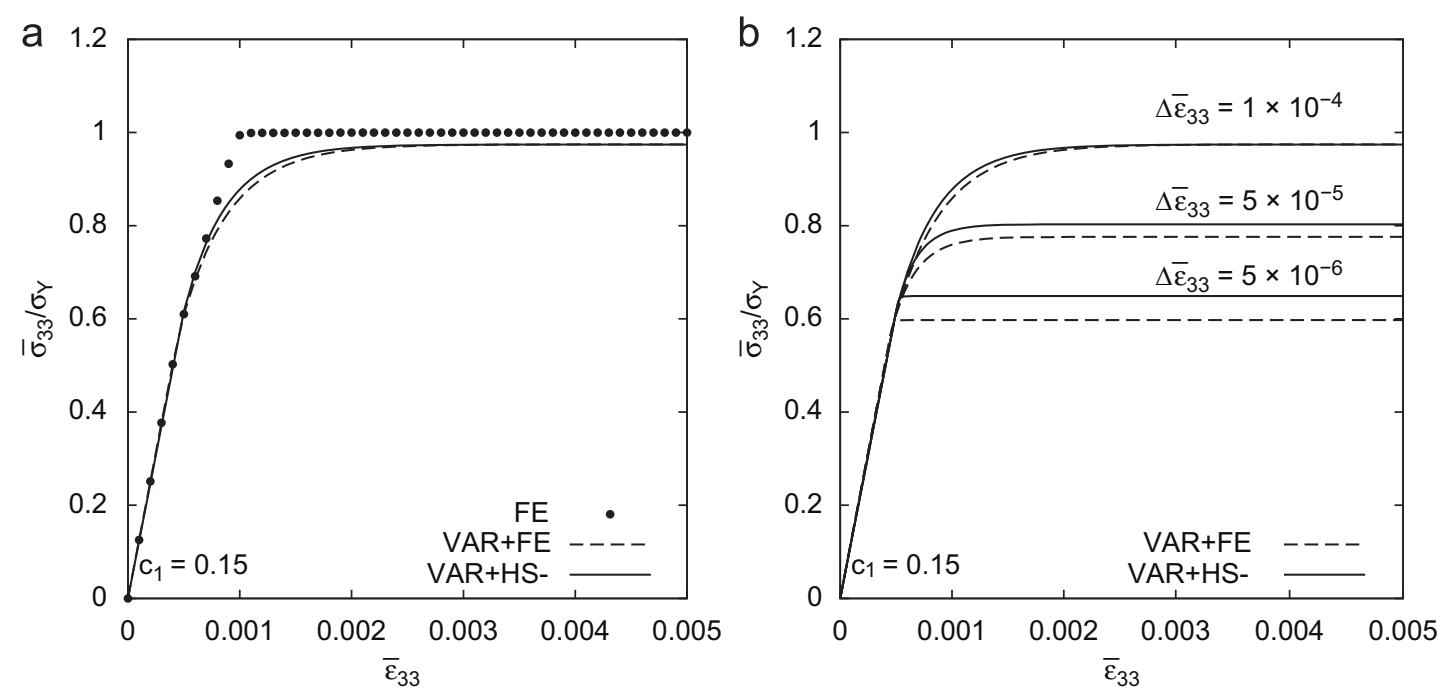

Fig. 10. (a) Macroscopic response of a periodic composite with a perfectly plastic matrix and perfectly plastic inclusions $\left(c_{1}=0.15\right)$. Both phases have the same yield stress. (b) The convergence of the VAR +FE and VAR + HS-models is assessed by considering successively 50,100 and 1000 strain increments to reach the final elongation.

\subsection{Perfectly plastic inclusions and matrix}

Finally, we consider the case of two perfectly plastic phases: $R^{(1)}(p)=R^{(2)}(p)=0$ with identical yield stresses but distinct elastic properties, identical to those considered before. The overall yield stress of the composite is obviously $\bar{\sigma}_{Y}=\sigma_{Y}^{(1)}=\sigma_{Y}^{(2)}$, and the heterogeneous elastic properties should affect only the macroscopic yield strain. Unfortunately, the variational procedure fails to predict the exact overall yield stress (Fig. 10(a)). Worse: a strong dependence on the number of loadsteps is observed, with the overall yield stress decreasing when the load increment is reduced (Fig. 10(b)). A look at the phase response (not shown) shows that the matrix stress never reaches the yield point, while the inclusion plastic strain is overestimated.

These results illustrate the limits of the proposed model in which plastic strain incompatibilities are neglected for the localization step. In the present example, inclusions are stiffer than the matrix and reach their yield point first. After several load increments, the average plastic strain update is observed to tend towards the macroscopic strain increment:

$$
\left\langle\Delta \varepsilon^{p}\right\rangle=c_{1}\left\langle\Delta \varepsilon^{p}\right\rangle_{1} \rightarrow \Delta \bar{\varepsilon} .
$$

As the (uniform) eigenstrain increases at the same rate as the macroscopic strain, moments of the trial strain in the phases (estimated by corresponding moments of the field $\boldsymbol{\varepsilon}^{\prime}=\boldsymbol{\varepsilon}-\left\langle\boldsymbol{\varepsilon}_{n}^{p}\right\rangle$ in the LCC, see relations (79)) reach a constant value, so as $\Delta p^{(r)}$ and $\mu_{0}^{(r)}$. Consequently, when this "regime" stage is reached, the phase and macroscopic stress tend to a constant value. Such behavior can be intuitively understood comparing (95) with a similar relation in homogeneous perfect plasticity, where

$$
\dot{\varepsilon}^{p} \rightarrow \dot{\varepsilon} \quad \text { or, incrementally } \Delta \varepsilon^{p} \rightarrow \Delta \varepsilon,
$$

from which it also follows that $\Delta \varepsilon^{\text {tr }} \rightarrow 0$. Only one load step in homogeneous, perfect plasticity suffices to reach the regime. In the composite, this number is higher (here, about 8), and does not seem to depend on the level of macroscopic strain. Consequently, the regime is reached at lower macroscopic strain when the load increment is reduced, causing the load step sensitivity shown in Fig. 10(b).

\section{Concluding remarks}

Section 4 presented an original equivalent formulation of the homogenization problem involving the potential of a thermoelastic composite in which the plastic strain field at the previous time step plays the role of an eigenstrain field. In Section 5, the composite mechanical response was estimated based on the following approximations: (i) piecewise uniform comparison moduli (and accumulated plastic strain as a consequence) and (ii) uniform reference plastic strain for $t_{n}$ for the whole composite. The first approximation is commonly adopted in variational procedures (like the variational procedure of Ponte Castañeda, 1991) and seems unavoidable. The second one amounts to neglect both intra- and inter-phase plastic strain incompatibilities when solving the localization problem on the LCC. Previous plastic strain is accounted for on average only. Despite this rough approximation, the estimate provides fairly good predictions in most examples tested so far in the context of two-phase particulate composites. A notable exception is the case of a composite 
with two perfectly plastic phases having the same yield stress (Section 6.3). In this case, neglecting inter-phase plastic incompatibilities leads to inconsistent results.

Important aspects of the proposed method are summarized:

- The model is designed for true elasto-plasticity, and does not require the approximation of visco-plasticity or perfect plasticity. One explicitly accounts for the existence of an elasticity domain, nonlinear hardening, and the hereditary behavior. In particular, arbitrary loading paths are handled.

- The formulation suggests an original localization rule for elasto-plastic composites based on "trial secant" operators computed for the second moments of the trial strain field. These operators are softer than the corresponding elastic ones.

- The algorithmic structure of the incremental equations of elasto-plasticity is preserved in the homogenization scheme. The model yields a homogenized yield criterion for each elasto-plastic phase, and a homogenized radial return equation for the internal variable. Both the homogenized yield criterion and the return mapping equation are based on the second-order moment of the trial strain field as a result from the variational procedure. A homogenized flow rule for per-phase averages of the plastic strain was also derived.

Future developments of the approach should focus on the account of inter-phase plastic strain incompatibilities in the localization problem. This can be achieved considering piecewise uniform reference plastic strain in the thermoelastic problem (70), instead of a uniform one. However, the definition of a proper uniform reference plastic strain for the phase is not straightforward (Lahellec and Suquet, 2007a; Brassart, 2011), and is left for future work.

\section{Acknowledgments}

L.B. and L.D. are mandated by the National Fund for Scientific Research (FNRS, Belgium). The authors thank the reviewer for a very constructive review which pointed out shortcomings in the initial manuscript, and $\mathrm{N}$. Lahellec for helpful discussions.

\section{Appendix A. Computation of the kinematic variable using Lagrange multipliers}

The kinematic variable $\boldsymbol{N}$ must minimize the functional $J_{\Delta}$ (23) under constraints (6). The corresponding Lagrangian functional reads

$$
\mathcal{L}\left(\boldsymbol{\varepsilon}_{n+1}, p_{n+1}, \boldsymbol{N}, \lambda_{1}, \lambda_{2}\right)=\frac{1}{2}\left(\varepsilon_{n+1}^{t r}-\Delta p \boldsymbol{N}\right): \boldsymbol{C}^{e}:\left(\boldsymbol{\varepsilon}_{n+1}^{t r}-\Delta p \boldsymbol{N}\right)+\psi^{p}\left(p_{n+1}\right)-\psi_{n}+\Delta t \phi\left(\frac{\Delta p}{\Delta t}\right)+\lambda_{1} \operatorname{tr}(\boldsymbol{N})+\lambda_{2}\left(\boldsymbol{N}: \boldsymbol{N}-\frac{3}{2}\right) .
$$

The kinematic variable $\boldsymbol{N}$ must satisfy the following condition:

$$
\frac{\partial \mathcal{L}}{\partial \boldsymbol{N}}\left(\boldsymbol{\varepsilon}_{n+1}, p_{n+1}, \boldsymbol{N}, \lambda_{1}, \lambda_{2}\right)=0,
$$

that is,

$$
0=-\Delta p \boldsymbol{C}^{e}:\left(\boldsymbol{\varepsilon}_{n+1}^{t r}-\Delta p \boldsymbol{N}\right)+\lambda_{1} \mathbf{1}+2 \lambda_{2} \boldsymbol{N}=-\Delta p\left(\boldsymbol{\sigma}_{n+1}^{t r}-\Delta p\left(\boldsymbol{C}^{e}: \boldsymbol{N}\right)\right)+\lambda_{1} \mathbf{1}+2 \lambda_{2} \boldsymbol{N} .
$$

Additional hypotheses about the free-energy function are required in order to determine $\boldsymbol{N}$. Here, isotropic elasticity is assumed, so that the elastic stiffness tensor may be decomposed into volumetric and deviatoric parts, see expression (32). In this case, condition (99) becomes

$$
-\Delta p\left(\boldsymbol{\sigma}_{n+1}^{t r}-2 \mu \Delta p \boldsymbol{N}\right)+\lambda_{1} \mathbf{1}+2 \lambda_{2} \boldsymbol{N}=0 .
$$

The trace of the above expression is computed, leading to

$$
\lambda_{1}=\frac{1}{3} \Delta p \operatorname{tr}\left(\boldsymbol{\sigma}_{n+1}^{t r}\right),
$$

which, introduced in (100) yields

$$
-\Delta p\left(\boldsymbol{\sigma}_{n+1}^{t r}-\frac{1}{3} \operatorname{tr}\left(\boldsymbol{\sigma}_{n+1}^{t r}\right)\right) \mathbf{1}+2 \mu(\Delta p)^{2} \boldsymbol{N}+2 \lambda_{2} \boldsymbol{N}=0,
$$

which can be rewritten as

$$
-\Delta p\left(\boldsymbol{s}_{n+1}^{t r}\right)=-2 \mu(\Delta p)^{2} \boldsymbol{N}-2 \lambda_{2} \boldsymbol{N} .
$$

This equation shows that the direction $\boldsymbol{N}$ is "aligned" with the tensor $\boldsymbol{s}_{n+1}^{t r}$. The normalizing condition in (6) finally gives

$$
\boldsymbol{N}=\frac{3}{2} \frac{\boldsymbol{s}_{n+1}^{t r}}{\sigma_{e q, n+1}^{t r}}
$$




\section{Appendix B. Link with the transformation field analysis (TFA)}

A straightforward simplification to problem (50) would be to restrict the space of solutions for the internal and kinematic variables to piecewise uniform fields:

$$
\Delta p(\boldsymbol{x})=\sum_{r=1}^{N} \chi^{(r)}(\boldsymbol{x}) \Delta p^{(r)}, \quad \mathbf{N}(\boldsymbol{x})=\sum_{r=1}^{N} \chi^{(r)}(\boldsymbol{x}) \mathbf{N}^{(r)},
$$

with $\operatorname{tr}\left(\boldsymbol{N}^{(r)}\right)=0$ and $\boldsymbol{N}^{(r)}: \boldsymbol{N}^{(r)}=3 / 2$. Consequently, the plastic strain tensor is also piecewise uniform:

$$
\boldsymbol{\varepsilon}^{p}(\boldsymbol{x})=\sum_{r=1}^{N} \chi^{(r)}(\boldsymbol{x}) \boldsymbol{\varepsilon}^{p(r)},
$$

its update being given by the following discretized flow rule:

$$
\Delta \boldsymbol{\varepsilon}^{p(r)}=\Delta p^{(r)} \mathbf{N}^{(r)} \text {. }
$$

Using the trial fields (105) in (50) leads to an upper bound for the effective incremental potential (indices $n+1$ are omitted for simplicity):

$$
\bar{W}_{\Delta}(\overline{\boldsymbol{\varepsilon}}) \leq \inf _{\boldsymbol{\varepsilon} \in \mathcal{K}(\overline{\boldsymbol{\varepsilon}})}\left\langle\inf _{\Delta p^{(s)}, \boldsymbol{N}^{(s)}}\left\{\sum_{r=1}^{N} \chi^{(r)}(\boldsymbol{x})\left\{\psi^{(r)}\left(\boldsymbol{\varepsilon}, \boldsymbol{\varepsilon}^{p(r)}, p^{(r)}\right)-\psi_{n}^{(r)}(\boldsymbol{x})+\Delta t \phi^{(r)}\left(\frac{\Delta p^{(r)}}{\Delta t}\right)\right\}\right\}\right\rangle .
$$

Permuting the order of the infimum operations over the strain field and the internal variables, the following alternative expression is obtained:

$$
\bar{W}_{\Delta}(\overline{\boldsymbol{\varepsilon}}) \leq \inf _{\Delta p^{(s)} \mathbf{N}^{(s)}}\left\{\bar{W}_{0}\left(\boldsymbol{\varepsilon}, \boldsymbol{\varepsilon}^{p(s)}\right)+\left\langle\sum_{r=1}^{N} \chi^{(r)}(\boldsymbol{x})\left(\psi^{p(r)}\left(p^{(r)}\right)-\psi_{n}^{(r)}(\boldsymbol{x})+\Delta t \phi^{(r)}\left(\frac{\Delta p^{(r)}}{\Delta t}\right)\right)\right\rangle\right\}
$$

where

$$
\bar{W}_{0}\left(\boldsymbol{\varepsilon}, \varepsilon^{p(s)}\right)=\inf _{\varepsilon \in \mathcal{K}(\overline{\boldsymbol{\varepsilon}})}\left\langle\sum_{r=1}^{N} \chi^{(r)}(\boldsymbol{x}) \frac{1}{2}\left(\boldsymbol{\varepsilon}-\boldsymbol{\varepsilon}^{p(r)}\right): \boldsymbol{C}^{e}:\left(\boldsymbol{\varepsilon}-\boldsymbol{\varepsilon}^{p(r)}\right)\right\rangle .
$$

Consequently, the local stress and strain fields may be determined from an elastic analysis on a thermoelastic composite in which the piecewise uniform plastic strain acts as an eigenstrain. This is exactly the assumption sustaining the TFA.

The stationarity condition w.r.t. $\Delta p^{(r)}$ in (109) yields the condition:

$$
\left\langle Y\left(\mathbf{N}^{(r)}, p^{(r)}\right)\right\rangle_{r}=\langle\boldsymbol{\sigma}\rangle_{r}: \boldsymbol{N}^{(r)}-R^{(r)}\left(p^{(r)}\right)=\left\langle\frac{\partial \phi^{(r)}}{\partial \dot{p}}\left(\frac{\Delta p}{\Delta t}\right)\right\rangle_{r} .
$$

The constrained minimization w.r.t. $\mathbf{N}^{(r)}$ is performed using Lagrange multipliers (see Appendix A), leading to

$$
\boldsymbol{N}^{(r)}=\frac{\left\langle\boldsymbol{e}-\boldsymbol{\varepsilon}_{n}^{p(r)}\right\rangle_{r}}{\left(\left\langle\boldsymbol{\varepsilon}-\boldsymbol{\varepsilon}_{n}^{(r)}\right\rangle_{r}\right)_{e q}} .
$$

Combining the last two equations, it is readily seen that $\Delta p^{(r)}$ obeys the radial return scheme driven by the first moment of the stress in phase $r$ of the thermoelastic composite.

\section{References}

ABAQUS 6.9, 2009. A General-purpose Finite Element Software. ABAQUS Inc., Pawtucket, RI, USA.

Benveniste, Y., 1987. A new approach to the application of Mori-Tanaka's theory in composite materials. Mech. Mater. 6, 147-157.

Berveiller, M., Zaoui, A., 1979. An extension of the self-consistent scheme to plastically-flowing polycrystals. J. Mech. Phys. Solids 26, 325-344.

Bobeth, M., Diener, G., 1986. Field fluctuations in multicomponent mixtures. J. Mech. Phys. Solids 34, 1-17.

Brassart, L., 2011. Homogenization of Elasto-(visco)plastic Composites: History-dependent Incremental and Variational Approaches. Ph.D. Thesis, Ecole Polytechnique de Louvain, Université catholique de Louvain. URL 〈http://dial.academielouvain.be/handle/boreal:75974 〉.

Buryachenko, V., 1999. Elastic-plastic behavior of elastically homogeneous materials with a random field of inclusions. Int. J. Plasticity 15, 687-720.

Carini, A., 1996. Colonnetti's minimum principle extension to generally non-linear materials. Int. J. Solids Struct. 33, 121-144.

Chaboche, J.-L., Kanouté, P., 2003. Sur les approximations "isotrope" et "anisotrope" de l'opérateur tangent pour les méthodes tangentes incrémentale et affine. C. R. Mécanique 331, 857-864.

Chaboche, J.L., Kanouté, P., Roos, A., 2005. On the capabilities of mean-field approaches for the description of plasticity in metal matrix composites. Int. J. Plasticity $21,1409-1434$.

Chaboche, J.-L., Kruch, S., Maire, J., Pottier, T., 2001. Towards a micromechanics based inelastic and damage modeling of composites. Int. J. Plasticity 17, 411-439.

Comi, C., Corigliano, A., Maier, G., 1991. Extremum properties of finite-step solutions in elastoplasticity with nonlinear hardening. Int. J. Solids Struct. 27, 965-981.

Doghri, I., 2000. Mechanics of Deformable Solids: Linear and Nonlinear, Analytical and Computational Aspects. Springer.

Doghri, I., Friebel, C., 2005. Effective elasto-plastic properties of inclusion-reinforced composites. Study of shape, orientation and cyclic response. Mech. Mater. 37, 45-68.

Doghri, I., Ouaar, A., 2003. Homogenization of two-phase elasto-plastic composite materials and structures: study of tangent operators, cyclic plasticity and numerical algorithms. Int. J. Solids Struct. 40, 1681-1712. 
Dvorak, G., 1992. Transformation field analysis of inelastic composite materials. Proc. R. Soc. London A 437, $311-327$.

Dvorak, G., Benveniste, Y., 1992. On transformation strains and uniform fields in multiphase elastic media. Proc. R. Soc. London A 437, 291-310.

Germain, P., Nguyen, Q., Suquet, P., 1983. Continuum thermodynamics. J. Appl. Mech. 50, 1010-1020.

Geuzaine, C., Remacle, J.-F., 2009. Gmsh: a three-dimensional finite element mesh generator with built-in pre- and post-processing facilities. Int. J. Numer. Meth. Eng. 79, 1309-1331.

Gilormini, P., 1995. Insuffisance de l'extension classique du modèle auto-cohérent au comportement non linéaire. C. R. Acad. Sci. Paris 320 (Série IIb), $115-122$.

González, C., LLorca, J., 2000. A self-consistent approach to the elasto-plastic behaviour of two-phase materials including damage. J. Mech. Phys. Solids 48, 675-692.

González, C., Segurado, J., LLorca, J., 2004. Numerical simulation of elasto-plastic deformation of composites: evolution of stress microfields and implications for homogenization models. J. Mech. Phys. Solids 52, 1573-1593.

Halphen, B., Nguyen, Q., 1975. Sur les matériaux standards généralisés. J. Méc. 14, 39-63.

Hashin, Z., Shtrikman, S., 1963. A variational approach to the theory of the elastic behavior of multiphase materials. J. Mech. Phys. Solids 11 , 127-140.

Hill, R., 1965a. Continuum micro-mechanics of elastoplastic polycrystals. J. Mech. Phys. Solids 13, 89-101.

Hill, R., 1965b. A self-consistent mechanics of composite materials. J. Mech. Phys. Solids 13, 213-222.

Hill, R., 1967. The essential structure of constitutive laws for metal composites and polycrystals. J. Mech. Phys. Solids 15, 79-95.

Hutchinson, J.W., 1970. Elastic-plastic behaviour of polycrystalline metals and composites. Proc. R. Soc. London A 319, $247-272$.

Hutchinson, J.W., 1976. Bounds and self-consistent estimates for creep of polycrystalline materials. Proc. R. Soc. London A 348, 101-127.

Kreher, W.S., 1990. Residual stresses and stored elastic energy of composites and polycrystals. J. Mech. Phys. Solids 38, 115-128.

Kröner, E., 1958. Berechnung der elastischen konstanten des vielkristalls aus den konstanten des einkristalls. Z. Phys. 151, 504-518.

Lahellec, N., Suquet, P., 2007a. On the effective behavior of nonlinear inelastic composites: I. Incremental variational principles. J. Mech. Phys. Solids 55, $1932-1963$.

Lahellec, N., Suquet, P., 2007b. On the effective behavior of nonlinear inelastic composites: II. A second-order procedure. J. Mech. Phys. Solids 55, 1964-1992.

Lebensohn, R., Tomé, C., 1993. A self-consistent anisotropic approach for the simulation of plastic deformation and texture development of polycrystals: application to zirconium alloys. Acta Metall. Mater. 41, 2611-2624.

Lemaître, J., Chaboche, J.-L., 1990. Mechanics of Solid Materials. Cambridge University Press.

Martin, J.B., Kaunda, M.A.E., Isted, R., 1996. Internal variable formulations of elastic-plastic dynamic problems. Int. J. Impact Eng. 18, 849-858.

Masson, R., Bornert, M., Suquet, P., Zaoui, A., 2000. An affine formulation for the prediction of the effective properties of nonlinear composites and polycrystals. J. Mech. Phys. Solids 48, 1203-1227.

Maugin, G.A., 1992. The Thermomechanics of Plasticity and Fracture. Cambridge University Press.

Mialon, P., 1986. Eléments d'analyse et de résolution numérique des relations de l'élasto-plasticité. EDF Bull. Dir. Etud. Rech. Sér. C 3, 57-89.

Michel, J., Suquet, P., 2003. Nonuniform transformation field analysis. Int. J. Solids Struct. 40, 6937-6955.

Miehe, C., 2002. Strain-driven homogenization of inelastic microstructures and composites based on an incremental variational formulation. Int. J. Numer. Meth. Eng. 55, 1285-1322.

Molinari, A., Canova, G., Azhi, S., 1987. A self-consistent approach to the large deformation polycrystal viscoplasticity. Acta Metall. Mater. 35, 2983-2994.

Moreau, J., 1976. Application of convex analysis to the treatment of elasto-plastic systems. in: Germain, P., Nayroles, B. (Eds.), Applications of Methods of Functional Analysis to Problems in Mechanics, Springer-Verlag.

Mori, T., Tanaka, K., 1973. Average stress in matrix and average elastic energy of materials with misfitting inclusions. Acta Metall. 21, 571-574.

Ortiz, M., Stainier, L., 1999. The variational formulation of viscoplastic constitutive updates. Comput. Methods Appl. Mech. Eng. 171, $419-444$.

Pierard, O., Doghri, I., 2006. Study of various estimates of the macroscopic tangent operator in the incremental homogenization of elastoplastic composites. Int. J. Multiscale Comput. Eng. 4, 521-543.

Pierard, O., Gonzalez, C., Segurado, J., LLorca, J., Doghri, I., 2007. Micromechanics of elasto-plastic materials reinforced with ellipsoidal inclusions. Int. J. Solids Struct. 44, 6945-6962.

Ponte Castañeda, P., 1991. The effective mechanical properties of nonlinear isotropic composites. J. Mech. Phys. Solids 39, 45-71.

Ponte Castañeda, P., 1992. New variational principles in plasticity and their application to composite materials. J. Mech. Phys. Solids 40, $1757-1788$.

Ponte Castañeda, P., 1996. Exact second-order estimates for the effective mechanical properties of nonlinear composite materials. J. Mech. Phys. Solids 44, $827-862$.

Ponte Castañeda, P., Suquet, P., 1998. Nonlinear composites. Adv. Appl. Mech. 34, 171-302.

Rekik, A., Auslender, F., Bornert, M., Zaoui, A., 2007. Objective evaluation of linearization procedures in nonlinear homogenization: a methodology and some implications on the accuracy of micromechanical schemes. Int. J. Solids Struct. 44, 3468-3496.

Rockafellar, R., 1970. Convex Analysis. Princeton University Press.

Segurado, J., LLorca, J., González, C., 2002. On the accuracy of mean-field approaches to simulate the plastic deformation of composites. Scr. Mater. 46, 525-529.

Simo, J.C., Hughes, T., 1998. Computational Inelasticity. Springer.

Suquet, P., 1993. Overall potentials and extremal surfaces of power law or ideally plastic materials. J. Mech. Phys. Solids 41, 981-1002.

Suquet, P., 1995. Overall properties of nonlinear composites: a modified secant moduli theory and its link with Ponte Castañeda's nonlinear variational procedure. C. R. Acad. Sci. Paris 320 (Série IIb), 563-571.

Suquet, P., 1996. Overall properties of nonlinear composites: remarks on secant and incremental formulations. in: Zaoui, A., Pineau, A. (Eds.), Micromechanics of Plasticity and Damage of Multiphase Materials, Kluwer Academic Publishers, Dordrecht, pp. 149-156.

Suquet, P., 1997. Effective properties of nonlinear composites. In: Suquet, P. (Ed.), Continuum Micromechanics. CISM Lecture Notes, vol. 377. , Springer Verlag, New York, pp. 197-264.

Tandon, G.P., Weng, G.J., 1988. A theory of particle-reinforced plasticity. J. Appl. Mech. 55, 126-135.

Turner, P., Tomé, C.N., 1994. A study of residual-stresses in Zircaloy-2 with rod texture. Acta Metall. Mater. 42, 4143-4153.

Wilkins, M., 1964. Calculation of elasto-plastic flow. in: Alder, B., et al. (Eds.), Methods of Computational Physics, Academic Press, New York.

Willis, J.R., 1977. Bounds and self-consistent estimates for the overall moduli of anisotropic composites. J. Mech. Phys. Solids 25, 185-202. 